\title{
A generalized penalty function in Sparre Andersen risk models with surplus-dependent premium
}

\author{
Eric C.K. Cheung*
}

January 1, 2011

\begin{abstract}
In a general Sparre Andersen risk model with surplus-dependent premium income, the generalization of Gerber-Shiu function proposed by Cheung et al. (2010a) is studied. A general expression for such Gerber-Shiu function is derived, and it is shown that its determination reduces to the evaluation of a transition function which is independent of the penalty function. Properties of and explicit expressions for such a transition function are derived when the surplus process is subject to (i) constant premium; (ii) a threshold dividend strategy; or (iii) credit interest. Extension of the approach is discussed for an absolute ruin model with debit interest.
\end{abstract}

Keywords: Generalized penalty function, Gerber-Shiu function, Sparre Andersen model, surplusdependent premium rate, threshold dividend strategy, credit interest, absolute ruin.

Acknowledgment: The author would like to thank the anonymous referee for his/her useful comments and suggestions which improved the paper. Support for Eric C.K. Cheung from a start-up fund provided by the Faculty of Science and the Department of Statistics and Actuarial Science at the University of Hong Kong is also gratefully acknowledged.

*Department of Statistics and Actuarial Science, University of Hong Kong, Pokfulam, Hong Kong. eckc@hku.hk 


\section{Introduction}

In this paper we model the surplus process of an insurance company as a generalized Sparre Andersen risk model with surplus-dependent premium rate. The motivation for a surplus-dependent premium rate is two-fold. First, as mentioned by Lin and Pavlova (2006), from the insurer's point of view, a higher surplus level allows the insurer to reduce premium to stay competitive. In contrast, in case of low surplus level, the insurer might need to charge a higher premium to avoid the possibility of insufficient funds. Second, from a mathematical point of view, the class of risk models with surplus-dependent premium rate includes a large variety of risk models which may involve dividend strategies and/or interest earnings, as we shall see later.

The model of interest is described as follows. Let $\left\{S_{t}\right\}_{t \geq 0} \equiv\left\{\sum_{i=1}^{N_{t}} Y_{i}\right\}_{t \geq 0}$ be the aggregate claims process, where the claim number process $\left\{N_{t}\right\}_{t \geq 0}$ is a renewal process defined through the sequence of independent and identically distributed (i.i.d.) positive interclaim times $\left\{V_{i}\right\}_{i=1}^{\infty}$, with $V_{1}$ being the time of the first claim and $V_{i}$ the time between the $(i-1)$-th claim and the $i$-th claim for $i=2,3, \ldots$. Let $K(t)=1-\bar{K}(t)=\operatorname{Pr}\{V \leq t\}$ be the cumulative distribution function (c.d.f.) of $V$, with $V$ being an arbitrary $V_{i}$. We further assume that $K(t)$ is differentiable and hence $V$ has density $k(t)=K^{\prime}(t)$ and Laplace transform $\widetilde{k}(s)=\int_{0}^{\infty} e^{-s t} k(t) d t$. Also, the claim size random variables (r.v.'s) $\left\{Y_{i}\right\}_{i=1}^{\infty}$, with $Y_{i}$ being the size of the $i$-th claim, are assumed to form a sequence of i.i.d. positive r.v.'s. In contrast to the traditional Sparre Andersen risk model in which the sequences $\left\{V_{i}\right\}_{i=1}^{\infty}$ and $\left\{Y_{i}\right\}_{i=1}^{\infty}$ are assumed to be independent, the generalized (or dependent) Sparre Andersen risk model relaxes this independence assumption by assuming that the pairs $\left\{\left(V_{i}, Y_{i}\right)\right\}_{i=1}^{\infty}$ are i.i.d. (see e.g. Albrecher and Teugels (2006) and Cheung et al. (2010b)). With $(V, Y)$ being an arbitrary pair of $\left(V_{i}, Y_{i}\right)$, it is convenient to specify the joint distribution of $(V, Y)$ by the product of the marginal density $k(t)$ and the conditional density of $Y$ given $V$. To do so, we define the conditional c.d.f. $P_{t}(y)=\operatorname{Pr}\{Y \leq y \mid V=t\}=1-\bar{P}_{t}(y)$ for $y \geq 0$. By assuming that $P_{t}(y)$ is differentiable in $y$ for each fixed $t>0$, its corresponding density is $p_{t}(y)=P_{t}^{\prime}(y)$, so that the joint density of $(V, Y)$ at $(t, y)$ is given by $p_{t}(y) k(t)$. We remark that the traditional Sparre Andersen model can be recovered from the present model by assuming that $P_{t}(y)$ does not depend on $t$. If we denote the surplus process of the insurance company by $\left\{U_{t}\right\}_{t \geq 0}$, then by surplus-dependent premium rate we mean that the instantaneous premium rate at time $t \geq 0$ is assumed to be $c\left(U_{t}\right)$, where $c($.$) is a positive function. Therefore \left\{U_{t}\right\}_{t \geq 0}$ satisfies

$$
d U_{t}=c\left(U_{t}\right) d t-d S_{t}, \quad t \geq 0
$$

We also assume the two technical conditions $\int_{0}^{x}[c(v)]^{-1} d v<\infty$ for any finite $x \geq 0$ and $\int_{0}^{\infty}[c(v)]^{-1} d v=$ $\infty$ (see Lin and Sendova (2008)). Indeed, the class of risk models with surplus-dependent premium rate includes many existing models as special cases. Apart from the simplest case of constant premium, it includes risk models with a threshold (Lin and Pavlova (2006) and Zhou (2004)) or a multi-threshold (Lin and Sendova (2008)) dividend strategy, and also risk models with credit interest (see e.g. Cai and Dickson (2002) and Sundt and Teugels (1995)) or even with liquid reserves (see e.g. Cai et al. (2009a) and Embrechts and Schmidli (1994)).

The main goal of this paper is the evaluation of the Gerber-Shiu function with a general penalty function in the generalized Sparre Andersen model, and this will be achieved by exploiting certain structural properties under a surplus-dependent premium rate. Here we give a brief review of the literature regarding the classical Gerber-Shiu functions. In Gerber and Shiu (1998), the expected discounted penalty 
function

$$
m_{\delta, 12}(u)=E\left[e^{-\delta T} w_{12}\left(U_{T^{-}},\left|U_{T}\right|\right) 1(T<\infty) \mid U_{0}=u\right], \quad u \geq 0
$$

was introduced, where $T$ is the time of ruin defined by $T=\inf \left\{t \geq 0: U_{t}<0\right\}$ with $T=\infty$ if $U_{t} \geq 0$ for all $t \geq 0$. In addition, $U_{T^{-}}$is the surplus immediately prior to ruin, $\left|U_{T}\right|$ is the deficit at ruin, $w_{12}(x, y)$ is the so-called penalty function, $1(A)$ is the indicator function of the event $A$, and $\delta \geq 0$ can either be viewed as a force of interest or a Laplace transform argument. The classical Gerber-Shiu function (1.1) (or its special cases) with $c(.) \equiv c$ has been studied extensively in the literature in various generalized Sparre Andersen models. For models assuming a specific dependency structure for the pair $(V, Y)$, interested readers are referred to Badescu et al. (2009), Boudreault et al. (2006) and Cossette et al. (2008). For an arbitrary dependency structure, the asymptotic ruin probability was studied by Albrecher and Teugels (2006).

In generalizing the Gerber-Shiu function (1.1), we shall incorporate an additional r.v. into the penalty function. With an initial surplus of $U_{0}=u$, we suppose that the first claim occurs at some time $t>0$. Furthermore, let $\gamma(u, s)$ denote the surplus level at time $s$ for $0 \leq s<t$, with the definition that $\gamma(u, t)=\gamma\left(u, t^{-}\right)$being the surplus level just before the first claim. Then,

$$
\gamma(u, t)=u+\int_{0}^{t} c(\gamma(u, s)) d s
$$

We define the sequence of $\left\{R_{n}\right\}_{n=0}^{\infty}$ recursively via the function $\gamma(u, t)$ such that $R_{0}=U_{0}=u$ and

$$
R_{n}=\gamma\left(R_{n-1}, V_{n}\right)-Y_{n}, \quad n=1,2, \ldots .
$$

Clearly, $R_{n}$ is the surplus level immediately following the $n$-th claim if $n \geq 1$, and therefore $R_{N_{T}-1}$ is the surplus immediately after the second last claim before ruin occurs if $N_{T}>1$, and $R_{N_{T}-1}=U_{0}=u$ if $N_{T}=1$. One could easily see that the definition $R_{0}=U_{0}=u$ is consistent with the usual assumption in a (generalized) Sparre Andersen model that a claim (called the 0-th claim) occurs at time $0^{-}$. Therefore in the case where ruin occurs upon the first claim, the second last claim can be regarded as the 0-th claim and thus the surplus immediately after the second last claim is $U_{0}$. Then (1.1) is generalized to

$$
m_{\delta}(u)=E\left[e^{-\delta T} w\left(U_{T^{-}},\left|U_{T}\right|, R_{N_{T}-1}\right) 1(T<\infty) \mid U_{0}=u\right], \quad u \geq 0,
$$

where $w(x, y, z)$ is the generalized penalty function. The generalized Gerber-Shiu function (1.3) was first introduced by Cheung et al. (2010a) in the context of a classical compound Poisson risk model with $c(.) \equiv c$, and Willmot and Woo (2010) generalized the study to the traditional Sparre Andersen model with Coxian interclaim times. In addition, Badescu et al. (2009) obtained the discounted joint density of the triplet $\left(U_{T^{-}},\left|U_{T}\right|, R_{N_{T}-1}\right)$ when $(V, Y)$ follows a bivariate phase-type distribution, and Cheung et al. (2010b) studied a more general Gerber-Shiu function than (1.3) by further incorporating the minimum surplus level before ruin in a general Sparre Andersen model. Obviously, the classical Gerber-Shiu function $m_{\delta, 12}(u)$ can be retrieved from $m_{\delta}(u)$ by letting $w(x, y, z) \equiv w_{12}(x, y)$.

We remark that Cheung et al. (2010a) showed that (1.3) can be used to study the last interclaim time before ruin $V_{N_{T}}=\left(U_{T^{-}}-R_{N_{T}-1}\right) / c$ (and its joint distribution with the claim causing ruin $Y_{N_{T}}=$ $\left.U_{T^{-}}+\left|U_{T}\right|\right)$ when $c(.) \equiv c$. When the premium rate is surplus-dependent, the last interclaim time $V_{N_{T}}$ can still be studied via (1.3) through the introduction of a new function as follows. With an initial surplus of $U_{0}=u$, if $x=\gamma(u, t)$ denotes the surplus level just before the first claim, it can be verified that the 
time of the first claim must be $t=\vartheta(u, x)$, where

$$
\vartheta(u, x)=\int_{u}^{x} \frac{d v}{c(v)}, \quad x>u .
$$

Then the last interclaim time can be expressed as $V_{N_{T}}=\vartheta\left(R_{N_{T}-1}, U_{T^{-}}\right)$. Interested readers are referred to Cheung et al. (2011) for some ordering properties of $V_{N_{T}}$ in relation to $V$ in the present model.

This paper is organized as follows. In Section 2, an expression for the generalized Gerber-Shiu function (1.3) is derived in terms of a transition function which is independent of the penalty function. Section 3 deals with the case with constant premium $c(.) \equiv c$, and the transition function characterizing $m_{\delta}(u)$ is studied in detail. More complicated assumptions on $c($.$) are studied in Sections 4$ and 5 respectively in which models with threshold dividend strategy or credit interest are covered. Extension of the approach is also discussed in the context of an absolute ruin model with debit interest in Section 6, and Section 7 ends the paper with some concluding remarks.

\section{The general structure}

As in Cheung et al. (2010a,b), we begin by introducing the joint distribution of the time of ruin $T$, the surplus prior to ruin $U_{T^{-}}$, the deficit at ruin $\left|U_{T}\right|$, and the surplus immediately after the second last claim before ruin $R_{N_{T}-1}$. According to the way the function $\vartheta(u, x)$ in (1.4) is defined, with an initial surplus of $U_{0}=u$, if ruin occurs on the first claim, there is a one-to-one relationship between $U_{T^{-}}$and $T$ given by $T=\vartheta\left(u, U_{T^{-}}\right)$, and additionally $R_{N_{T}-1}=u$. Thus, it is sufficient to specify the joint distribution of $\left(U_{T^{-}},\left|U_{T}\right|\right)$ at $(x, y)$ for ruin upon the first claim. In order to have a deficit of $\left|U_{T}\right|=y$ after reaching level $U_{T^{-}}=x$, the first claim has to be of size $x+y$. By applying the joint density of $\left(V_{1}, Y_{1}\right)$ (with a change of variable), such joint (defective) density of $\left(U_{T^{-}},\left|U_{T}\right|\right)$ is given by

$$
h_{1}(x, y \mid u)=\frac{1}{c(x)} k(\vartheta(u, x)) p_{\vartheta(u, x)}(x+y), \quad x>u ; y>0 .
$$

On the other hand, if ruin occurs on claims subsequent to the first, $T$ and $R_{N_{T}-1}$ are no longer simple functions of $U_{T^{-}}$and $\left|U_{T}\right|$, and we denote the joint (defective) density of $\left(T, U_{T^{-}},\left|U_{T}\right|, R_{N_{T}-1}\right)$ at $(t, x, y, z)$ given $U_{0}=u$ by $h_{2}(t, x, y, z \mid u)$. Then the discounted joint densities corresponding to $h_{1}(x, y \mid u)$ and $h_{2}(t, x, y, z \mid u)$ are given by

$$
h_{1, \delta}(x, y \mid u)=e^{-\delta \vartheta(u, x)} h_{1}(x, y \mid u), \quad x>u ; y>0,
$$

and

$$
h_{2, \delta}(x, y, z \mid u)=\int_{0}^{\infty} e^{-\delta t} h_{2}(t, x, y, z \mid u) d t, \quad x>z>0 ; y>0,
$$

respectively. Using the Dirac delta function $\Delta(x)$ heuristically defined as

$$
\Delta(x)= \begin{cases}+\infty, & x=0 \\ 0, & x \neq 0\end{cases}
$$

satisfying $\int_{-\infty}^{+\infty} \Delta(x) d x=1$, the discounted densities (2.2) and (2.3) can be expressed as

$$
\begin{aligned}
h_{1, \delta}(x, y \mid u) & =E\left[e^{-\delta T} \Delta\left(U_{T^{-}}-x\right) \Delta\left(\left|U_{T}\right|-y\right) 1\left(N_{T}=1\right) \mid U_{0}=u\right] \\
& =E\left[e^{-\delta V_{1}} \Delta\left(\gamma\left(u, V_{1}\right)-x\right) \Delta\left(Y_{1}-(x+y)\right)\right], \quad x>u ; y>0,
\end{aligned}
$$


and

$$
h_{2, \delta}(x, y, z \mid u)=E\left[e^{-\delta T} \Delta\left(U_{T^{-}}-x\right) \Delta\left(\left|U_{T}\right|-y\right) \Delta\left(R_{N_{T}-1}-z\right) 1\left(N_{T}>1\right) \mid U_{0}=u\right], x>z>0 ; y>0,
$$

respectively. We have the following lemma.

Lemma 1 The discounted densities $h_{1, \delta}$ and $h_{2, \delta}$ are related by

$$
h_{2, \delta}(x, y, z \mid u)=\tau_{\delta}(u, z) h_{1, \delta}(x, y \mid z), \quad x>z>0 ; y>0,
$$

where

$$
\tau_{\delta}(u, z)=\sum_{n=1}^{\infty} E\left[e^{-\delta \sum_{j=1}^{n} V_{j}} \Delta\left(R_{n}-z\right) 1\left(R_{i} \geq 0, i=1,2, \ldots, n\right) \mid U_{0}=u\right], \quad z>0
$$

Proof. By conditioning on the number of claims causing ruin, (2.5) can be expressed as

$$
\begin{aligned}
h_{2, \delta}(x, y, z \mid u)= & \sum_{n=1}^{\infty} E\left[e^{-\delta T} \Delta\left(U_{T^{-}}-x\right) \Delta\left(\left|U_{T}\right|-y\right) \Delta\left(R_{N_{T}-1}-z\right) 1\left(N_{T}=n+1\right) \mid U_{0}=u\right] \\
= & \sum_{n=1}^{\infty} E\left[\begin{array}{c}
e^{-\delta \sum_{j=1}^{n+1} V_{j}} \Delta\left(\gamma\left(R_{n}, V_{n+1}\right)-x\right) \Delta\left(\left|\gamma\left(R_{n}, V_{n+1}\right)-Y_{n+1}\right|-y\right) \\
\Delta\left(R_{n}-z\right) 1\left(N_{T}=n+1\right)
\end{array} \mid U_{0}=u\right] \\
= & \sum_{n=1}^{\infty} E\left[\begin{array}{c}
e^{-\delta \sum_{j=1}^{n+1} V_{j}} \Delta\left(\gamma\left(z, V_{n+1}\right)-x\right) \Delta\left(Y_{n+1}-(x+y)\right) \Delta\left(R_{n}-z\right) \\
1\left(R_{i} \geq 0, i=1,2, \ldots, n\right)
\end{array} \mid U_{0}=u\right] \\
= & \sum_{n=1}^{\infty} E\left[e^{\left.-\delta \sum_{j=1}^{n} V_{j} \Delta\left(R_{n}-z\right) 1\left(R_{i} \geq 0, i=1,2, \ldots, n\right) \mid U_{0}=u\right]}\right. \\
& \times E\left[e^{\left.-\delta V_{n+1} \Delta\left(\gamma\left(z, V_{n+1}\right)-x\right) \Delta\left(Y_{n+1}-(x+y)\right)\right], \quad x>z>0 ; y>0,}\right.
\end{aligned}
$$

where the last line follows from the independence of $\left(V_{n+1}, Y_{n+1}\right)$ on $\left\{\left(V_{i}, Y_{i}\right)\right\}_{i=1}^{n}$ together with the fact that for $n=1,2, \ldots, R_{n}$ only depends on $\left\{\left(V_{i}, Y_{i}\right)\right\}_{i=1}^{n}$ and $R_{0}=U_{0}$. By further using the fact that $\left(V_{n+1}, Y_{n+1}\right)$ has identical distribution as $\left(V_{1}, Y_{1}\right)$, application of (2.4) to (2.8) leads to $(2.6)$ with $\tau_{\delta}(u, z)$ given by (2.7).

The result given in Lemma 1 is in fact very intuitive. In order for ruin to occur upon at least two claims and the surplus level after the second last claim before ruin to be $z$ (as $h_{2, \delta}(x, y, z \mid u)$ suggests), the surplus process $\left\{U_{t}\right\}_{t \geq 0}$, starting with initial surplus $U_{0}=u$, has to first make a transition from level $u$ to level $z$ after an arbitrary number $(\geq 1)$ of claims without ruin occurring in the interim. Such a transition is explained by the term $\tau_{\delta}(u, z)$. After reaching level $z$, the process restarts, and if the next claim causes ruin (according to $\left.h_{1, \delta}(x, y \mid z)\right)$, then the triplet $\left(U_{T^{-}},\left|U_{T}\right|, R_{N_{T}-1}\right)$ will be exactly $(x, y, z)$. An immediate consequence of Lemma 1 is the following Proposition.

Proposition 1 The generalized Gerber-Shiu function $m_{\delta}(u)$ in (1.3) has the representation

$$
m_{\delta}(u)=\beta_{\delta}(u)+\int_{0}^{\infty} \tau_{\delta}(u, z) \beta_{\delta}(z) d z, \quad u \geq 0,
$$

where

$$
\beta_{\delta}(u)=\int_{0}^{\infty} \int_{u}^{\infty} w(x, y, u) h_{1, \delta}(x, y \mid u) d x d y, \quad u \geq 0
$$


Proof. Since the generalized Gerber-Shiu function (1.3) is an expectation of a discounted penalty, it can simply be written as an integral of the penalty function with respect to the discounted densities $h_{1, \delta}$ and $h_{2, \delta}$ as

$$
\begin{aligned}
m_{\delta}(u) & =\int_{0}^{\infty} \int_{u}^{\infty} w(x, y, u) h_{1, \delta}(x, y \mid u) d x d y+\int_{0}^{\infty} \int_{0}^{\infty} \int_{z}^{\infty} w(x, y, z) h_{2, \delta}(x, y, z \mid u) d x d y d z \\
& =\int_{0}^{\infty} \int_{u}^{\infty} w(x, y, u) h_{1, \delta}(x, y \mid u) d x d y+\int_{0}^{\infty} \tau_{\delta}(u, z) \int_{0}^{\infty} \int_{z}^{\infty} w(x, y, z) h_{1, \delta}(x, y \mid z) d x d y d z \\
& =\beta_{\delta}(u)+\int_{0}^{\infty} \tau_{\delta}(u, z) \beta_{\delta}(z) d z
\end{aligned}
$$

where the second last line follows from the substitution of $(2.6)$, and $\beta_{\delta}(u)$ is as defined in (2.10).

Proposition 1 is again an intuitive result. Essentially, it separates the contributions to $m_{\delta}(u)$ into cases where ruin occurs on the first claim or on subsequent claims. To see this, it is clear from (2.10) that $\beta_{\delta}(u)$ is the contribution by ruin upon the first claim. For the remaining contribution from ruin on subsequent claims, the surplus process $\left\{U_{t}\right\}_{t \geq 0}$ has to first make a transition from level $u$ to an arbitrary level $z>0$ after a number of claims avoiding ruin enroute, which is represented by $\tau_{\delta}(u, z)$. Being at level $z$, ruin occurs on the next claim, giving rise to $\beta_{\delta}(z)$. Since the level $z$ is arbitrary, this explains the integral term $\int_{0}^{\infty} \tau_{\delta}(u, z) \beta_{\delta}(z) d z$.

Note that the representation (2.9) in Proposition 1 holds true very generally, as it has been mentioned in the introduction that the model with surplus-dependent premium considered here contains various risk models under dividend strategies or credit interest. The advantage of such representation is that the dependence of $m_{\delta}(u)$ on the penalty function $w(x, y, z)$ only appears through $\beta_{\delta}(u)$, which is explicitly given by (2.10) (since $h_{1, \delta}$ is known from (2.1) and (2.2)). Therefore, the generalized Gerber-Shiu function $m_{\delta}(u)$ can be characterized by the transition function $\tau_{\delta}(u, z)$ defined by $(2.7)$, which is independent of the choice of $w(x, y, z)$. Once $\tau_{\delta}(u, z)$ is determined, $m_{\delta}(u)$ follows accordingly. Thus, the determination of $m_{\delta}(u)$ reduces to the evaluation of $\tau_{\delta}(u, z)$.

Since the quantity $\tau_{\delta}(u, z)$ characterizes both the discounted joint density $h_{2, \delta}(x, y, z \mid u)$ (via $(2.6)$ ) and the generalized Gerber-Shiu function $m_{\delta}(u)$ (via (2.9)), the procedure of its determination is outlined here. The determination of $\tau_{\delta}(u, z)$ can be done through its Laplace transform defined by

$$
\varphi_{\delta, r}(u)=\int_{0}^{\infty} e^{-r z} \tau_{\delta}(u, z) d z, \quad u \geq 0
$$

which by substitution of (2.7) yields

$$
\varphi_{\delta, r}(u)=\sum_{n=1}^{\infty} E\left[e^{-\delta \sum_{j=1}^{n} V_{j}} e^{-r R_{n}} 1\left(R_{i} \geq 0, i=1,2, \ldots, n\right) \mid U_{0}=u\right], \quad u \geq 0 .
$$

Then, $\varphi_{\delta, r}(u)$ can obtained by conditioning on the time $t$ and the amount $y$ of the first claim as

$$
\varphi_{\delta, r}(u)=\int_{0}^{\infty} e^{-\delta t}\left\{\int_{0}^{\gamma(u, t)}\left[e^{-r(\gamma(u, t)-y)}+\varphi_{\delta, r}(\gamma(u, t)-y)\right] p_{t}(y) d y\right\} k(t) d t .
$$

Note that there is only contribution to $\varphi_{\delta, r}(u)$ if the first claim does not cause ruin. In such case, the term $e^{-r(\gamma(u, t)-y)}$ is due to the process reaching level $\gamma(u, t)-y$ immediately after the first claim, while $\varphi_{\delta, r}(\gamma(u, t)-y)$ represents the future contribution to $\varphi_{\delta, r}(u)$ with the process restarting at level $\gamma(u, t)-y$. 
Remark 1 It appears that the representation of $\varphi_{\delta, r}(u)$ given by $(2.12)$ has certain similarities with the so-called expected present value of total claim costs up to the time of ruin studied by Cai et al. (2009b) (see also Feng (2009)). According to Cai et al. (2009b, Equation (1.2)), it is given by (adopted to our notation)

$$
C(u)=E\left[\sum_{n=1}^{N_{T}} e^{-\delta W_{n}} \omega\left(U_{W_{n}^{-}}, U_{W_{n}}\right) \mid U_{0}=u\right], \quad u \geq 0,
$$

where $W_{n}=\sum_{j=1}^{n} V_{j}$ is the time of the $n$-th claim for $n=1,2, \ldots$, and $\omega(x, y)$ is a function representing the 'cost' associated to a claim. It can be verified that $\varphi_{\delta, r}(u)$ is indeed a special case of $C(u)$ under the choice

$$
\omega(x, y)=\left\{\begin{array}{ll}
e^{-r y}, & y \geq 0 \\
0, & y<0
\end{array} .\right.
$$

However, it is instructive to note that Cai et al. (2009b) and Feng (2009) defined $C(u)$ in the compound Poisson model and the Sparre Andersen model with phase-type interclaim times respectively, whereas in the present paper $\varphi_{\delta, r}(u)$ is defined more generally in a generalized Sparre Andersen risk model.

Nonetheless, in the simplest case of the classical compound Poisson risk model where $k(t)=\lambda e^{-\lambda t}$ and $p_{t}(y)=p(y)$, Cai et al. (2009b, Proposition 3.1) showed that $C(u)$ defined by (2.14) is a special case of the so-called expected present value of total operating costs up to default, namely

$$
H(u)=E\left[\int_{0}^{T} e^{-\delta t} l\left(U_{t}\right) \mid U_{0}=u\right], \quad u \geq 0,
$$

under the choice of 'cost function'

$$
l(x)=\lambda \int_{0}^{\infty} \omega(x, x-y) p(y) d y, \quad x \geq 0 .
$$

Hence, according to $(2.15)$, the function $\varphi_{\delta, r}(u)$ can be retrieved from (2.16) by letting

$$
l(x)=\lambda \int_{0}^{x} e^{-r(x-y)} p(y) d y, \quad x \geq 0 .
$$

We also refer interested readers to Feng (2009, Theorem 4.1) for the relationship between $C(u)$ and $H(u)$ in a Sparre Andersen model with phase-type interclaim times.

The integral equation (2.13) is usually solved by making additional distributional assumptions on the claim size and/or the interclaim time. In contrast, if we condition on the time and the amount of the first claim to get an integral equation for $m_{\delta}(u)$, we do not expect that such integral equation can be solved easily without making any further assumption on the form and/or differentiability of the penalty function $w(x, y, z)$ in case where $c($.$) is not constant. To illustrate the generality of the our approach,$ the remainder of this paper provides a detailed study of the function $\varphi_{\delta, r}(u)$ (and hence $\tau_{\delta}(u, z)$ ) under a variety of cases where specific choices of $c($.$) are assumed.$

We remark that in cases where $c($.$) is constant, (2.6) and (2.9) have been shown to hold true (with$ $\tau_{\delta}(u, z)$ obtained explicitly) in certain models which are special cases of the generalized Sparre Andersen risk model presented here. We refer interested readers to Badescu et al. (2009), Cheung et al. (2010a), Willmot and Woo (2010) and Woo (2010) for such details. Furthermore, equations of the form (2.6) and (2.9) also hold true in a matrix form in a semi-Markovian risk model with exponential interclaim time distribution. See Cheung and Landriault (2009). 


\section{Model with constant premium rate}

For this entire section, we make the assumption that $c(.) \equiv c$. Under such simplest premium rate function, we are able to provide a general form of the function $\tau_{\delta}(u, z)$ in terms of $\tau_{\delta}(0, z)$. As mentioned in Section 2 , the generalized Gerber-Shiu function $m_{\delta}(u)$ can be characterized by $\tau_{\delta}(u, z)$. Here we are going to analyze $\tau_{\delta}(u, z)$ through its Laplace transform $\varphi_{\delta, r}(u)$ defined by $(2.11)$.

To begin, define

$$
\phi_{\delta}=\int_{0}^{\infty} \int_{0}^{\infty}\left\{h_{1, \delta}(x, y \mid 0)+\int_{0}^{x} h_{2, \delta}(x, y, z \mid 0) d z\right\} d x d y
$$

and the (proper) ladder height density

$$
f_{\delta}(y)=\frac{1}{\phi_{\delta}} \int_{0}^{\infty}\left\{h_{1, \delta}(x, y \mid 0)+\int_{0}^{x} h_{2, \delta}(x, y, z \mid 0) d z\right\} d x, \quad y>0 .
$$

In general, $\phi_{\delta}<1$ if $\delta>0$ or the positive security loading condition $c E[V]>E[Y]$ holds. Note that $\phi_{\delta}$ and $f_{\delta}(y)$ only depend on the (defective) density of the deficit at ruin $\left|U_{T}\right|$ given $U_{0}=0$, which is already known in various Sparre Andersen risk models with or without dependency (see Examples 1 and 5).

By conditioning on the first drop in surplus below its initial level, one finds, according to (2.12),

$$
\varphi_{\delta, r}(u)=\varphi_{\delta, r}(0) e^{-r u}+\phi_{\delta} \int_{0}^{u} e^{-r(u-y)} f_{\delta}(y) d y+\phi_{\delta} \int_{0}^{u} \varphi_{\delta, r}(u-y) f_{\delta}(y) d y, \quad u \geq 0
$$

The derivation of (3.3) is given probabilistically as follows by noting that every claim could possibly contribute to $\varphi_{\delta, r}(u)$ as long as ruin has not occurred by the time of the claim arrival.

1. $\varphi_{\delta, r}(0) e^{-r u}$ is the contribution to $\varphi_{\delta, r}(u)$ by claims preceding the one causing the first drop in surplus (excluding the drop itself). The term $e^{-r u}$ appears because in considering the first drop the surplus process has been shifted by $u$ units.

2. $\phi_{\delta} \int_{0}^{u} e^{-r(u-y)} f_{\delta}(y) d y$ corresponds to the contribution to $\varphi_{\delta, r}(u)$ by the claim causing the first drop in surplus to some level $u-y(0<y<u)$.

3. $\phi_{\delta} \int_{0}^{u} \varphi_{\delta, r}(u-y) f_{\delta}(y) d y$ corresponds to the future contribution to $\varphi_{\delta, r}(u)$ if the first drop in surplus brings the surplus to some level $u-y(0<y<u)$.

The defective renewal equation (3.3) has solution (see e.g. Resnick (1992, Section 3.5))

$\varphi_{\delta, r}(u)=\varphi_{\delta, r}(0) e^{-r u}+\phi_{\delta}\left(e^{-r \cdot} * f_{\delta}\right)(u)+\frac{1}{1-\phi_{\delta}}\left[\varphi_{\delta, r}(0)\left(e^{-r \cdot} * g_{\delta}\right)(u)+\phi_{\delta}\left(e^{-r \cdot} * f_{\delta} * g_{\delta}\right)(u)\right], \quad u \geq 0$

where ' $*$ ' is the convolution operator defined by $\left(A_{1} * A_{2}\right)(x)=\int_{0}^{x} A_{1}(x-y) A_{2}(y) d y$ for two functions $A_{1}($.$) and A_{2}($.$) on (0, \infty)$, and $g_{\delta}(y)$ is the compound geometric density

$$
g_{\delta}(y)=\sum_{n=1}^{\infty}\left(1-\phi_{\delta}\right) \phi_{\delta}^{n} f_{\delta}^{* n}(y), \quad y>0
$$


with $f_{\delta}^{* n}($.$) being the n$-fold convolution of $f_{\delta}($.$) with itself. Clearly, the Laplace transform of g_{\delta}($.$) ,$ namely $\widetilde{g}_{\delta}(s)=\int_{0}^{\infty} e^{-s y} g_{\delta}(y) d y$, is given by

$$
\widetilde{g}_{\delta}(s)=\sum_{n=1}^{\infty}\left(1-\phi_{\delta}\right) \phi_{\delta}^{n}\left[\widetilde{f}_{\delta}(s)\right]^{n}
$$

where $\widetilde{f}_{\delta}(s)=\int_{0}^{\infty} e^{-s y} f_{\delta}(y) d y$. To simplify the term $\left(e^{-r \cdot} * f_{\delta} * g_{\delta}\right)(u)$ in (3.4), we consider

$$
\widetilde{f}_{\delta}(s) \widetilde{g}_{\delta}(s)=\sum_{n=1}^{\infty}\left(1-\phi_{\delta}\right) \phi_{\delta}^{n}\left[\widetilde{f}_{\delta}(s)\right]^{n+1}=\frac{1}{\phi_{\delta}} \widetilde{g}_{\delta}(s)-\left(1-\phi_{\delta}\right) \widetilde{f}_{\delta}(s),
$$

and hence

$$
\left(f_{\delta} * g_{\delta}\right)(u)=\frac{1}{\phi_{\delta}} g_{\delta}(u)-\left(1-\phi_{\delta}\right) f_{\delta}(u) .
$$

Thus, application of (3.6) to (3.4) followed by further simplifications leads to

$$
\varphi_{\delta, r}(u)=\varphi_{\delta, r}(0) e^{-r u}+\frac{1}{1-\phi_{\delta}}\left[\varphi_{\delta, r}(0)\left(e^{-r \cdot} * g_{\delta}\right)(u)+\left(e^{-r \cdot} * g_{\delta}\right)(u)\right], \quad u \geq 0 .
$$

We then aim at inverting (3.7) with respect to $r$ to obtain $\tau_{\delta}(u, z)$. By recalling that $\varphi_{\delta, r}(0)=$ $\int_{0}^{\infty} e^{-r z} \tau_{\delta}(0, z) d z$, we arrive at

$$
\varphi_{\delta, r}(0) e^{-r u}=\left\{\int_{0}^{\infty} e^{-r z} \tau_{\delta}(0, z) d z\right\} e^{-r u}=\int_{u}^{\infty} e^{-r z} \tau_{\delta}(0, z-u) d z
$$

and

$$
\begin{aligned}
\varphi_{\delta, r}(0)\left(e^{-r \cdot} * g_{\delta}\right)(u) & =\left\{\int_{0}^{\infty} e^{-r z} \tau_{\delta}(0, z) d z\right\}\left\{\int_{0}^{u} e^{-r x} g_{\delta}(u-x) d x\right\} \\
& =\int_{0}^{u} \int_{x}^{\infty} e^{-r z} \tau_{\delta}(0, z-x) g_{\delta}(u-x) d z d x \\
& =\left(\int_{0}^{u} \int_{0}^{z}+\int_{u}^{\infty} \int_{0}^{u}\right) e^{-r z} \tau_{\delta}(0, z-x) g_{\delta}(u-x) d x d z,
\end{aligned}
$$

and therefore inversion of (3.7) yields

$$
\tau_{\delta}(u, z)=\left\{\begin{array}{ll}
\frac{1}{1-\phi_{\delta}}\left\{g_{\delta}(u-z)+\int_{0}^{z} \tau_{\delta}(0, z-x) g_{\delta}(u-x) d x\right\}, & z<u \\
\tau_{\delta}(0, z-u)+\frac{1}{1-\phi_{\delta}} \int_{0}^{u} \tau_{\delta}(0, z-x) g_{\delta}(u-x) d x, & z \geq u
\end{array},\right.
$$

which relates $\tau_{\delta}(u, z)$ to $\tau_{\delta}(0, z)$. Relationship in the form of (3.8) can also be found in Woo (2010, Chapter 2.2.1). Note from (3.1), (3.2) and (3.5) that $\phi_{\delta}$ and $g_{\delta}(y)$ appearing in (3.8) can be expressed in terms of $h_{1, \delta}(x, y \mid 0)$ (which is known explicitly) and $h_{2, \delta}(x, y, z \mid 0)$ (which can be expressed in terms of $\tau_{\delta}(0, z)$ by $(2.6)$ in Lemma 1$)$. Therefore, in principle $\tau_{\delta}(0, z)$ is sufficient to characterize $\tau_{\delta}(u, z)$ and hence $m_{\delta}(u)$.

The next two examples illustrate the applications of $(2.13)$ to determine $\varphi_{\delta, r}(0)=\int_{0}^{\infty} e^{-r z} \tau_{\delta}(0, z) d z$ when either the interclaim time or the claim size is exponentially distributed.

Example 1 (Compound Poisson risk model) 
In this example we assume a classical compound Poisson risk model, i.e. $k(t)=\lambda e^{-\lambda t}$ and $p_{t}(y)=p(y)$. Then the integral equation (2.13) becomes

$$
\varphi_{\delta, r}(u)=\int_{0}^{\infty} \lambda e^{-(\lambda+\delta) t}\left\{\int_{0}^{u+c t}\left[e^{-r(u+c t-y)}+\varphi_{\delta, r}(u+c t-y)\right] p(y) d y\right\} d t .
$$

A change of variable results in

$$
\varphi_{\delta, r}(u)=\frac{\lambda}{c} \int_{u}^{\infty} e^{-\frac{\lambda+\delta}{c}(x-u)}\left\{\int_{0}^{x} e^{-r(x-y)} p(y) d y+\int_{0}^{x} \varphi_{\delta, r}(x-y) p(y) d y\right\} d x .
$$

Differentiation of the above equation with respect to $u$ results in the integro-differential equation

$$
\varphi_{\delta, r}^{\prime}(u)=\frac{\lambda+\delta}{c} \varphi_{\delta, r}(u)-\frac{\lambda}{c} \int_{0}^{u} e^{-r(u-y)} p(y) d y-\frac{\lambda}{c} \int_{0}^{u} \varphi_{\delta, r}(u-y) p(y) d y .
$$

We remark that the above equation can also be obtained using (2.17) in Remark 1 along with Cai et al. (2009b, Theorem 3.1). Taking Laplace transform on both sides of (3.9) yields

$$
s \widetilde{\varphi}_{\delta, r}(s)-\varphi_{\delta, r}(0)=\frac{\lambda+\delta}{c} \widetilde{\varphi}_{\delta, r}(s)-\frac{\lambda}{c} \widetilde{p}(s) \frac{1}{r+s}-\frac{\lambda}{c} \widetilde{p}(s) \widetilde{\varphi}_{\delta, r}(s) .
$$

where $\widetilde{\varphi}_{\delta, r}(s)=\int_{0}^{\infty} e^{-s u} \varphi_{\delta, r}(u) d u$ and $\widetilde{p}(s)=\int_{0}^{\infty} e^{-s y} p(y) d y$. Rearranging terms in the above equation leads to

$$
\left[s-\frac{\lambda+\delta}{c}+\frac{\lambda}{c} \widetilde{p}(s)\right] \widetilde{\varphi}_{\delta, r}(s)=\varphi_{\delta, r}(0)-\frac{\lambda}{c} \widetilde{p}(s) \frac{1}{r+s} .
$$

By letting $\rho=\rho(\delta)$ be the unique non-negative solution to the Lundberg's fundamental equation (in $\xi$ )

$$
\xi-\frac{\lambda+\delta}{c}+\frac{\lambda}{c} \widetilde{p}(\xi)=0
$$

with $\widetilde{\varphi}_{\delta, r}(\rho)<\infty$ putting $s=\rho$ into (3.10) yields

$$
\varphi_{\delta, r}(0)=\frac{\lambda}{c} \widetilde{p}(\rho) \frac{1}{r+\rho} .
$$

Inversion with respect to $r$ in $(3.12)$ gives

$$
\tau_{\delta}(0, z)=\frac{\lambda}{c} \widetilde{p}(\rho) e^{-\rho z}, \quad z>0
$$

We remark that (3.8) together with (3.13) lead to the same representation of $\tau_{\delta}(u, z)$ given in Cheung et al. (2010a, Theorem 2.2). In addition, in this case $\phi_{\delta}$ and $f_{\delta}(y)$ are given by (see Gerber and Shiu (1998))

$$
\phi_{\delta}=\frac{\lambda}{c} \int_{0}^{\infty} \int_{0}^{\infty} e^{-\rho x} p(x+y) d x d y=\frac{\lambda}{c} \mathcal{T}_{0} \mathcal{T}_{\rho} p(0)
$$

and

$$
f_{\delta}(y)=\frac{\int_{0}^{\infty} e^{-\rho x} p(x+y) d x}{\int_{0}^{\infty} \int_{0}^{\infty} e^{-\rho x} p(x+y) d x d y}=\frac{\mathcal{T}_{\rho} p(y)}{\mathcal{T}_{0} \mathcal{T}_{\rho} p(0)}, \quad y>0,
$$

respectively. Here the Dickson-Hipp operator $\mathcal{T}_{s}$ (see Dickson and Hipp (2001)) is defined as, for any integrable function $A($.$) on (0, \infty)$ and any complex number $s$ with $\operatorname{Re}(s) \geq 0$,

$$
\mathcal{T}_{s} A(y)=\int_{y}^{\infty} e^{-s(x-y)} A(x) d x, \quad y \geq 0 .
$$


These expressions will be useful in Section 4.

We remark that $\varphi_{\delta, r}(0)$ can also be obtained in a similar manner as in Example 1 for more complicated interclaim time distribution such as the Coxian distribution. Inversion with respect to $r$ again results in $\tau_{\delta}(0, z)$ and hence $\tau_{\delta}(u, z)$ can be characterized via (3.8). The function $\tau_{\delta}(u, z)$ under the assumption that the interclaim times are Coxian was derived by Willmot and Woo (2010) using a different method.

Example 2 (Sparre Andersen risk model with arbitrary interclaim times and exponential claims)

In this example we assume a traditional Sparre Andersen risk model with exponential claims, i.e. $p_{t}(y)=p(y)=\mu e^{-\mu y}$. By taking Laplace transforms on both sides of (3.3) and solving for $\widetilde{\varphi}_{\delta, r}(s)$, one finds

$$
\widetilde{\varphi}_{\delta, r}(s)=\frac{\varphi_{\delta, r}(0)+\phi_{\delta} \widetilde{f}_{\delta}(s)}{(r+s)\left[1-\phi_{\delta} \widetilde{f}_{\delta}(s)\right]} .
$$

It is known from Willmot (2007, Example 3.1) that with exponential claim sizes, the ladder height density defined by (3.2) is also the same exponential density, and therefore the above equation can be rewritten as

$$
\widetilde{\varphi}_{\delta, r}(s)=\frac{\varphi_{\delta, r}(0)(\mu+s)+\phi_{\delta} \mu}{(r+s)\left[\mu\left(1-\phi_{\delta}\right)+s\right]} .
$$

By defining $\kappa=\kappa(\delta)=\mu\left(1-\phi_{\delta}\right)$, it is clear that $\widetilde{\varphi}_{\delta, r}(s)$ can be resolved into partial fractions as, for some constants $A_{1}$ and $A_{2}$,

$$
\widetilde{\varphi}_{\delta, r}(s)=\frac{A_{1}}{s+\kappa}+\frac{A_{2}}{s+r},
$$

which, upon inversion, yields

$$
\varphi_{\delta, r}(u)=A_{1} e^{-\kappa u}+A_{2} e^{-r u}, \quad u \geq 0 .
$$

Meanwhile, under exponential claim size assumption, the integral equation (2.13) reduces to

$$
\varphi_{\delta, r}(u)=\int_{0}^{\infty} e^{-\delta t}\left\{\int_{0}^{u+c t}\left[e^{-r(u+c t-y)}+\varphi_{\delta, r}(u+c t-y)\right] \mu e^{-\mu y} d y\right\} k(t) d t .
$$

Then, the solution form (3.16) is substituted into both sides of the above equation. We omit the algebra and arrive at

$$
\begin{aligned}
A_{1} e^{-\kappa u}+A_{2} e^{-r u}= & A_{1} \frac{\mu}{\mu-\kappa} \widetilde{k}(\delta+c \kappa) e^{-\kappa u}+\left(1+A_{2}\right) \frac{\mu}{\mu-r} \widetilde{k}(\delta+c r) e^{-r u} \\
& -\left[A_{1} \frac{\mu}{\mu-\kappa}+\left(1+A_{2}\right) \frac{\mu}{\mu-r}\right] \widetilde{k}(\delta+c \mu) e^{-\mu u}
\end{aligned}
$$

Since the above equation holds true for all $u \geq 0$, equating the coefficients of $e^{-\kappa u}, e^{-r u}$ and $e^{-\mu u}$ on both sides leads to

$$
\left\{\begin{array}{l}
1=\frac{\mu}{\mu-\kappa} \widetilde{k}(\delta+c \kappa) \\
A_{2}=\left(1+A_{2}\right) \frac{\mu}{\mu-r} \widetilde{k}(\delta+c r) \\
A_{1} \frac{\mu}{\mu-\kappa}+\left(1+A_{2}\right) \frac{\mu}{\mu-r}=0
\end{array} .\right.
$$

The first equation of (3.17) is equivalent to saying that $-\kappa$ is the unique negative root of the Lundberg's fundamental equation (in $\xi$ )

$$
1=\frac{\mu}{\mu+\xi} \widetilde{k}(\delta-c \xi)
$$


See e.g. Landriault and Willmot (2008). Solving the remaining two equations in (3.17) for $A_{1}$ and $A_{2}$ followed by substitution into (3.16) gives

$$
\varphi_{\delta, r}(u)=\frac{1}{r-\mu[1-\widetilde{k}(\delta+c r)]}\left[(\mu-\kappa) e^{-\kappa u}-\mu \widetilde{k}(\delta+c r) e^{-r u}\right], \quad u \geq 0 .
$$

which characterizes $\tau_{\delta}(u, z)$ via its Laplace transform with respect to $z$.

Example 3 (Explicit calculations under exponential-exponential assumptions)

This example aims at finding the generalized Gerber-Shiu function $m_{\delta}(u)$ under the choice of penalty function $w(x, y, z)=e^{-s_{1} x-s_{2} y-s_{3} z}$, so that $m_{\delta}(u)$ represents the joint Laplace transform of the quadruple $\left(T, U_{T^{-}},\left|U_{T}\right|, R_{N_{T}-1}\right)$ with transform arguments $\left(\delta, s_{1}, s_{2}, s_{3}\right)$. For an explicit evaluation, we assume the classical compound Poisson risk model with exponential claims, i.e. $k(t)=\lambda e^{-\lambda t}$ and $p_{t}(y)=p(y)=$ $\mu e^{-\mu y}$, so that the notations defined in both Examples 1 and 2 can be used here. In particular, $\rho \geq 0$ and $-\kappa<0$ are the roots of the equivalent equations (3.11) and (3.18) which are now quadratic equations. First, we proceed by evaluating $\tau_{\delta}(u, z)$ via (3.8). Since $\rho$ satisfies (3.11), (3.13) becomes

$$
\tau_{\delta}(0, z)=\left(\frac{\lambda+\delta}{c}-\rho\right) e^{-\rho z}=(\mu-\kappa) e^{-\rho z}, \quad z>0
$$

where the last equality follows because the sum of roots of (3.11), namely $\rho-\kappa$, equals $(\lambda+\delta) / c-\mu$. Moreover, the compound geometric density $g_{\delta}(y)$ can be obtained by differentiating equation (5.38) of Gerber and Shiu (1998), which gives rise to

$$
g_{\delta}(y)=\phi_{\delta} \kappa e^{-\kappa y}, \quad y>0 .
$$

By substituting (3.20) and (3.21) into (3.8), performing straightforward integration and using the fact that $\kappa=\mu\left(1-\phi_{\delta}\right)$, we arrive at

$$
\tau_{\delta}(u, z)=\left\{\begin{array}{ll}
\frac{\mu-\kappa}{\rho+\kappa} e^{-\kappa u}\left[(\mu+\rho) e^{\kappa z}-(\mu-\kappa) e^{-\rho z}\right], & z<u \\
\frac{\mu-\kappa}{\rho+\kappa} e^{-\rho z}\left[(\mu+\rho) e^{\rho u}-(\mu-\kappa) e^{-\kappa u}\right], & z \geq u
\end{array} .\right.
$$

Next, we shall find $\beta_{\delta}(u)$ defined by (2.10). With both $Y$ and $V$ exponentially distributed, (2.2) reduces to

$$
h_{1, \delta}(x, y \mid u)=\frac{\lambda \mu}{c} e^{-\frac{\lambda+\delta}{c}(x-u)-\mu(x+y)}, \quad x>u ; y>0 .
$$

Hence, under the penalty function $w(x, y, z)=e^{-s_{1} x-s_{2} y-s_{3} z},(2.10)$ is found to be

$$
\beta_{\delta}(u)=\frac{\frac{\lambda \mu}{c}}{\left(s_{1}+\frac{\lambda+\delta}{c}+\mu\right)\left(s_{2}+\mu\right)} e^{-\left(s_{1}+s_{3}+\mu\right) u}, \quad u \geq 0 .
$$

Substitution of (3.22) and (3.23) into (2.9) followed by some manipulations leads to

$$
\begin{aligned}
m_{\delta}(u)= & \frac{\lambda \mu}{c} \frac{s_{1}+s_{3}+\frac{\lambda+\delta}{c}+\mu}{\left(s_{1}+\frac{\lambda+\delta}{c}+\mu\right)\left(s_{2}+\mu\right)\left(s_{1}+s_{3}+\mu+\rho\right)\left(s_{1}+s_{3}+\mu-\kappa\right)} \\
& \times\left[\left(s_{1}+s_{3}\right) e^{-\left(s_{1}+s_{3}+\mu\right) u}+(\mu-\kappa) e^{-\kappa u}\right], \quad u \geq 0,
\end{aligned}
$$

which agrees with Cheung et al. (2010a, Equation (66)). 


\section{Model with a threshold dividend strategy}

In this section, we shall consider the general Sparre Andersen risk model under a threshold dividend strategy. Under a threshold strategy, it is assumed that the insurance company receives actual premium at rate $c$. Moreover, there is a fixed level $b>0$, called the threshold level, such that whenever the surplus process is above $b$ and ruin has not occurred, dividend is paid at rate $\alpha>0$ to the shareholders, otherwise no dividend is paid. The positive security loading condition under the threshold model is $\alpha<c-E[Y] / E[V]$, which will be assumed here. For further details regarding this model, interested readers are referred to Gerber and Shiu (2006), Lin and Pavlova (2006) and Zhou (2004). In our setting, the threshold model is recovered by the choice of $c($.$) given by$

$$
c(u)=\left\{\begin{array}{ll}
c_{1}=c, & 0 \leq u \leq b \\
c_{2}=c-\alpha, & u>b
\end{array} .\right.
$$

To emphasize the dependence of $\varphi_{\delta, r}(u)$ (and $\tau_{\delta}(u, z)$ ) on the threshold level $b$, we shall write $\varphi_{\delta, r}(u ; b)$ instead of $\varphi_{\delta, r}(u)$ with $\varphi_{\delta, r}(u ; b)=\int_{0}^{\infty} e^{-r z} \tau_{\delta}(u, z ; b) d z$. Also, in general $\varphi_{\delta, r}(u ; b)$ is of different functional form depending on whether $0 \leq u \leq b$ or $u>b$, and therefore we shall use the notation

$$
\varphi_{\delta, r}(u ; b)=\left\{\begin{array}{ll}
\varphi_{\delta, r, 1}(u ; b)=\int_{0}^{\infty} e^{-r z} \tau_{\delta, 1}(u, z ; b) d z, & 0 \leq u \leq b \\
\varphi_{\delta, r, 2}(u ; b)=\int_{0}^{\infty} e^{-r z} \tau_{\delta, 2}(u, z ; b) d z, & u>b
\end{array} .\right.
$$

By continuity one also has

$$
\varphi_{\delta, r, 1}(b ; b)=\varphi_{\delta, r, 2}\left(b^{+} ; b\right),
$$

so one can easily extend the domain of $\varphi_{\delta, r, 2}(u ; b)$ to include $u=b$.

For later use, we define, for $i=1,2$, the corresponding $\varphi_{\delta, r}$ function in a (threshold-free) model with $c(.) \equiv c_{i}$ as $\varphi_{\delta, r, i}(u)=\int_{0}^{\infty} e^{-r z} \tau_{\delta, i}(u, z) d z$. Then we let $\phi_{\delta, i}$ and $f_{\delta, i}($.$) be identical to \phi_{\delta}$ and $f_{\delta}($.$) given$ by (3.1) and (3.2) respectively but evaluated with $c$ replaced by $c_{i}$. Furthermore, $g_{\delta, i}($.$) is the compound$ geometric density (3.5) with $\phi_{\delta, i}$ and $f_{\delta, i}($.$) in place of \phi_{\delta}$ and $f_{\delta}($.$) respectively. Clearly, \varphi_{\delta, r, i}(u)$ has solution given by (3.7) with $\phi_{\delta, i}$ and $g_{\delta, i}($.$) in place of \phi_{\delta}$ and $g_{\delta}($.$) .$

Analogous to the way (3.3) is obtained, the same argument leads to

$$
\begin{aligned}
\varphi_{\delta, r, 2}(u ; b)= & \varphi_{\delta, r, 2}(0) e^{-r u}+\phi_{\delta, 2} \int_{0}^{u} e^{-r(u-y)} f_{\delta, 2}(y) d y+\phi_{\delta, 2} \int_{0}^{u-b} \varphi_{\delta, r, 2}(u-y ; b) f_{\delta, 2}(y) d y \\
& +\phi_{\delta, 2} \int_{u-b}^{u} \varphi_{\delta, r, 1}(u-y ; b) f_{\delta, 2}(y) d y, \quad u \geq b
\end{aligned}
$$

which, upon defining $\zeta_{\delta, r, b}(u)=\varphi_{\delta, r, 2}(u+b ; b)$ for $u \geq 0$, can be rewritten as

$$
\zeta_{\delta, r, b}(u)=\phi_{\delta, 2} \int_{0}^{u} \zeta_{\delta, r, b}(u-y) f_{\delta, 2}(y) d y+\varpi_{\delta, r}(u+b ; b), \quad u \geq 0
$$

where

$$
\varpi_{\delta, r}(u ; b)=\phi_{\delta, 2} \int_{u-b}^{u} \varphi_{\delta, r, 1}(u-y ; b) f_{\delta, 2}(y) d y+\varphi_{\delta, r, 2}(0) e^{-r u}+\phi_{\delta, 2} \int_{0}^{u} e^{-r(u-y)} f_{\delta, 2}(y) d y, \quad u \geq b .
$$


Clearly, (4.3) is a defective renewal equation in $\zeta_{\delta, r, b}(u)$ with solution

$$
\zeta_{\delta, r, b}(u)=\frac{1}{1-\phi_{\delta, 2}} \int_{0}^{u} \varpi_{\delta, r}(u+b-y ; b) g_{\delta, 2}(y) d y+\varpi_{\delta, r}(u+b ; b), \quad u \geq 0,
$$

and therefore $\varphi_{\delta, r, 2}(u ; b)$ can be expressed as

$$
\varphi_{\delta, r, 2}(u ; b)=\frac{1}{1-\phi_{\delta, 2}} \int_{0}^{u-b} \varpi_{\delta, r}(u-y ; b) g_{\delta, 2}(y) d y+\varpi_{\delta, r}(u ; b), \quad u \geq b .
$$

Note that in the representation $(4.5) \varphi_{\delta, r, 2}(u ; b)$ is expressed in terms of $\varpi_{\delta, r}(u ; b)$ which in turn depends on $\varphi_{\delta, r, 1}(u ; b)$ via (4.4). The next example illustrates the determination of $\varphi_{\delta, r, 1}(u ; b)$ and hence $\tau_{\delta, 1}(u, z ; b)$ and $\tau_{\delta, 2}(u, z ; b)$ in the compound Poisson risk model.

\section{Example 4 (Compound Poisson risk model)}

By assuming a classical compound Poisson risk model, i.e. $k(t)=\lambda e^{-\lambda t}$ and $p_{t}(y)=p(y)$, we omit the rather straightforward details and state that the integral equation (2.13) implies

$$
\varphi_{\delta, r, 1}^{\prime}(u ; b)=\frac{\lambda+\delta}{c_{1}} \varphi_{\delta, r, 1}(u ; b)-\frac{\lambda}{c_{1}} \int_{0}^{u} e^{-r(u-y)} p(y) d y-\frac{\lambda}{c_{1}} \int_{0}^{u} \varphi_{\delta, r, 1}(u-y ; b) p(y) d y, \quad 0 \leq u \leq b,
$$

and

$$
\begin{aligned}
\varphi_{\delta, r, 2}^{\prime}(u ; b)= & \frac{\lambda+\delta}{c_{2}} \varphi_{\delta, r, 2}(u ; b)-\frac{\lambda}{c_{2}} \int_{0}^{u} e^{-r(u-y)} p(y) d y \\
& -\frac{\lambda}{c_{2}}\left\{\int_{0}^{u-b} \varphi_{\delta, r, 2}(u-y ; b) p(y) d y+\int_{u-b}^{u} \varphi_{\delta, r, 1}(u-y ; b) p(y) d y\right\}, \quad u \geq b .
\end{aligned}
$$

We only need to turn our attention to the integro-differential equation (4.6) since one can verify that (see Lin and Pavlova (2006, Theorem 4.1)) (4.7) can indeed be transformed to (4.2) with solution (4.5). Note that (4.6) is structurally identical to (3.9) except for the domain. Therefore, from the theory of integro-differential equations (see e.g. Lakshmikantham and Rao (1995, p.50)), we have that

$$
\varphi_{\delta, r, 1}(u ; b)=\varphi_{\delta, r, 1}(u)+\eta_{\delta, r}(b) v_{\delta}(u), \quad 0 \leq u \leq b,
$$

for some constant $\eta_{\delta, r}(b)$ independent of $u$, and $v_{\delta}(u)$ is a solution to the homogeneous version of the integro-differential equation (3.9) and is unique up to a multiplicative constant. By Cheung and Landriault (2010), the solution with initial condition $v_{\delta}(0)=1$ can be expressed as

$$
v_{\delta}(u)=e^{\rho_{1} u}+\frac{1}{1-\phi_{\delta, 1}} \int_{0}^{u} e^{\rho_{1}(u-y)} g_{\delta, 1}(y) d y, \quad u \geq 0 .
$$

Here, for $i=1,2, \rho_{i}$ is the unique non-negative root to the Lundberg's fundamental equation (3.11) with $c$ being replaced by $c_{i}$. Then $\phi_{\delta, i}$ and $f_{\delta, i}(y)$ (and hence $\left.g_{\delta, i}(y)\right)$ can correspondingly be evaluated via (3.14) and (3.15). We remark that an alternative form for $v_{\delta}(u)$ was also given in Bühlmann (1970, Section 6.4.9).

With the application of (4.4), $\eta_{\delta, r}(b)$ can then be determined by (4.5), (4.8) and the continuity condition (4.1) as

$$
\eta_{\delta, r}(b)=\frac{\phi_{\delta, 2} \int_{0}^{b} \varphi_{\delta, r, 1}(b-y) f_{\delta, 2}(y) d y-\varphi_{\delta, r, 1}(b)+\varphi_{\delta, r, 2}(0) e^{-r b}+\phi_{\delta, 2} \int_{0}^{b} e^{-r(b-y)} f_{\delta, 2}(y) d y}{v_{\delta}(b)-\phi_{\delta, 2} \int_{0}^{b} v_{\delta}(b-y) f_{\delta, 2}(y) d y} .
$$


Now we already have a full characterization of $\varphi_{\delta, r, 1}(u ; b)$ and $\varphi_{\delta, r, 2}(u ; b)$ via (4.8) and (4.5).

In order to invert (4.8) and (4.5) with respect to $r$ to obtain $\tau_{\delta, 1}(u, z ; b)$ and $\tau_{\delta, 2}(u, z ; b)$, note that with the use of (3.12) one can write

$$
\begin{aligned}
\varphi_{\delta, r, 2}(0) e^{-r u}+\phi_{\delta, 2} \int_{0}^{u} e^{-r(u-y)} f_{\delta, 2}(y) d y & =\frac{\lambda}{c_{2}} \widetilde{p}\left(\rho_{2}\right) \frac{1}{r+\rho_{2}} e^{-r u}+\phi_{\delta, 2} \int_{0}^{u} e^{-r y} f_{\delta, 2}(u-y) d y \\
& =\frac{\lambda}{c_{2}} \widetilde{p}\left(\rho_{2}\right) \int_{u}^{\infty} e^{-r y} e^{-\rho_{2}(y-u)} d y+\phi_{\delta, 2} \int_{0}^{u} e^{-r y} f_{\delta, 2}(u-y) d y \\
& =\int_{0}^{\infty} e^{-r z} \chi_{\delta}(u, z) d z
\end{aligned}
$$

where

$$
\chi_{\delta}(u, z)=\left\{\begin{array}{ll}
\phi_{\delta, 2} f_{\delta, 2}(u-z), & z<u \\
\frac{\lambda}{c_{2}} \widetilde{p}\left(\rho_{2}\right) e^{-\rho_{2}(z-u)}, & z \geq u
\end{array} .\right.
$$

Using (4.9) and (4.10), inversion of (4.8) with respect to $r$ leads to

$$
\tau_{\delta, 1}(u, z ; b)=\tau_{\delta, 1}(u, z)+\frac{\phi_{\delta, 2} \int_{0}^{b} \tau_{\delta, 1}(b-y, z) f_{\delta, 2}(y) d y-\tau_{\delta, 1}(b, z)+\chi_{\delta}(b, z)}{v_{\delta}(b)-\phi_{\delta, 2} \int_{0}^{b} v_{\delta}(b-y) f_{\delta, 2}(y) d y} v_{\delta}(u), \quad 0 \leq u \leq b .
$$

Similarly, by application of (4.10) again, (4.4) can be written as

$$
\varpi_{\delta, r}(u ; b)=\int_{0}^{\infty} e^{-r z} \sigma_{\delta}(u, z ; b) d z, \quad u \geq b,
$$

where

$$
\sigma_{\delta}(u, z ; b)=\phi_{\delta, 2} \int_{u-b}^{u} \tau_{\delta, 1}(u-y, z ; b) f_{\delta, 2}(y) d y+\chi_{\delta}(u, z), \quad u \geq b .
$$

Hence, upon substitution of (4.12), inversion of (4.5) with respect to $r$ yields

$$
\tau_{\delta, 2}(u, z ; b)=\frac{1}{1-\phi_{\delta, 2}} \int_{0}^{u-b} \sigma_{\delta}(u-y, z ; b) g_{\delta, 2}(y) d y+\sigma_{\delta}(u, z ; b), \quad u \geq b .
$$

To summarize this example, for $0 \leq u \leq b, \tau_{\delta, 1}(u, z ; b)$ is explicitly given by (4.11), while for $u \geq b$, $\tau_{\delta, 2}(u, z ; b)$ is given by (4.14). It is instructive to note that the expression (4.14) involves $\sigma_{\delta}(u, z ; b)$, which depends on $\tau_{\delta, 1}(u, z ; b)$ via $(4.13)$.

We remark that a more general interclaim time than in the previous example such as a generalized Erlang- $n$ distribution can also be used. In such case, the corresponding $\phi_{\delta, 2}$ and ladder height density $f_{\delta, 2}(y)$ can be obtained from equation (8.3) of Gerber and Shiu (2005) so as to make use of the defective renewal equation (4.3). In addition, equation in the form of (4.8) also holds true but with $\eta_{\delta, r}(b) v_{\delta}(u)$ replaced by $\sum_{i=1}^{n} \eta_{\delta, r, i}(b) v_{\delta, i}(u)$, where the $n$ linearly independent homogeneous solutions $\left\{v_{\delta, i}(u)\right\}_{i=1}^{n}$ were studied by Li and Garrido (2004, Section 4) and the constants $\left\{\eta_{\delta, r}(b)\right\}_{i=1}^{n}$ are to be determined by the appropriate boundary conditions. Similar ideas apply for certain models involving dependency as well (see Example 5). Furthermore, the methodology presented in this section can be adopted to the case where the risk process is subject to a multi-threshold dividend strategy (see Lin and Sendova (2008)).

So far the first four examples are concerned with Sparre Andersen models in which the generic r.v.'s $Y$ and $V$ are independent. To illustrate the generality of our approach, in the next example we shall 
show how to determine $\varphi_{\delta, r}(u ; b)$ in the dependency model by Boudreault et al. (2006) under a threshold strategy.

Example 5 (Boudreault et al. (2006)'s dependency model)

In this model, it is assumed that $k(t)=\lambda e^{-\lambda t}$ and

$$
p_{t}(y)=e^{-\varsigma t} p_{1}(y)+\left(1-e^{-\varsigma t}\right) p_{2}(y), \quad t, y>0,
$$

where $p_{i}($.$) is a proper density function for i=1,2$ and $\varsigma>0$ is a dependence parameter. In parallel to (4.6) and (4.7), one finds that the integral equation (2.13) can be reduced to the piecewise integrodifferential equation (in $u$ ) as

$$
\begin{aligned}
& \left(\frac{\lambda+\delta+\varsigma}{c_{1}} \mathcal{I}-\mathcal{D}\right)\left(\frac{\lambda+\delta}{c_{1}} \mathcal{I}-\mathcal{D}\right) \varphi_{\delta, r}(u ; b)=\frac{\lambda}{c_{1}}\left(\frac{\lambda+\delta}{c_{1}} \mathcal{I}-\mathcal{D}\right)\left\{\int_{0}^{u} \varphi_{\delta, r}(u-y ; b) p_{1}(y) d y+\varrho_{r, 1}(u)\right\} \\
& +\frac{\lambda}{c_{1}} \frac{\varsigma}{c_{1}}\left\{\int_{0}^{u} \varphi_{\delta, r}(u-y ; b) p_{2}(y) d y+\varrho_{r, 2}(u)\right\}, \quad 0 \leq u \leq b,
\end{aligned}
$$

and

$$
\begin{aligned}
\left(\frac{\lambda+\delta+\varsigma}{c_{2}} \mathcal{I}-\mathcal{D}\right)\left(\frac{\lambda+\delta}{c_{2}} \mathcal{I}-\mathcal{D}\right) \varphi_{\delta, r}(u ; b)= & \frac{\lambda}{c_{2}}\left(\frac{\lambda+\delta}{c_{2}} \mathcal{I}-\mathcal{D}\right)\left\{\int_{0}^{u} \varphi_{\delta, r}(u-y ; b) p_{1}(y) d y+\varrho_{r, 1}(u)\right\} \\
& +\frac{\lambda}{c_{2}} \frac{\varsigma}{c_{2}}\left\{\int_{0}^{u} \varphi_{\delta, r}(u-y ; b) p_{2}(y) d y+\varrho_{r, 2}(u)\right\}, \quad u \geq b
\end{aligned}
$$

where for $i=1,2$,

$$
\varrho_{r, i}(u)=\int_{0}^{u} e^{-r(u-y)} p_{i}(y) d y, \quad u \geq 0,
$$

and $\mathcal{I}$ and $\mathcal{D}$ represent the identity and differentiation operators respectively. Similar to Example 4, (4.16) can be transformed to (4.2) and thus $\varphi_{\delta, r, 2}(u ; b)$ is given by (4.5). Here $\phi_{\delta, 2}$ and $f_{\delta, 2}(y)$ (and hence $\left.g_{\delta, 2}(y)\right)$ are available from Boudreault et al. (2006, Theorem 5), whereas $\varphi_{\delta, r, 2}(0)=\int_{0}^{\infty} e^{-r z} \tau_{\delta, 2}(0, z) d z$ appearing via $\varpi_{\delta, r}(u ; b)$ can be obtained from Woo (2010, Chapter 3.1.5). Moreover, (4.15) has solution

$$
\varphi_{\delta, r, 1}(u ; b)=\varphi_{\delta, r, 1}(u)+\sum_{i=1}^{2} \eta_{\delta, r, i}(b) v_{\delta, i}(u), \quad 0 \leq u \leq b,
$$

where $\varphi_{\delta, r, 1}(u)=\int_{0}^{\infty} e^{-r z} \tau_{\delta, 1}(u, z) d z$ is again provided by Woo (2010, Chapter 3.1.5), and $\left\{v_{\delta, i}(u)\right\}_{i=1}^{2}$ are the linearly independent solutions to the homogeneous version of (4.15) with extended domain $u \geq 0$ and are given by Landriault (2008). In addition, the constants $\left\{\eta_{\delta, r}(b)\right\}_{i=1}^{2}$ can be solved from the system of two linear equations consisting of the continuity condition (4.1) and the additional condition

$$
c_{1} \varphi_{\delta, r, 1}^{\prime}(b ; b)=c_{2} \varphi_{\delta, r, 2}^{\prime}(b ; b) .
$$

We remark that the above condition suggests that in general $\varphi_{\delta, r}^{\prime}(u ; b)$ is not continuous at $u=b$, and it appeared in the literature in the study of Gerber-Shiu functions under threshold strategies. See Gerber and Shiu (2006, Section 10) and Lin and Pavlova (2006, Section 3).

Having obtained $\left\{\eta_{\delta, r}(b)\right\}_{i=1}^{2}$, the quantities $\varphi_{\delta, r, 1}(u ; b)$ and $\varphi_{\delta, r, 2}(u ; b)$ are fully characterized by (4.17) and (4.5). Inversion of Laplace transforms with respect to $r$ yields $\tau_{\delta, 1}(u, z ; b)$ and $\tau_{\delta, 2}(u, z ; b)$. Since the ideas involved are identical to those in Example 4, the straightforward but rather tedious algebraic details are omitted here. 


\section{$5 \quad$ Model with credit interest}

In a risk model with credit interest, it is assumed that the insurance company receives actual premium income at a constant rate $c>0$, while at the same time interest is received on its surplus at a constant force of interest $\varepsilon>0$. Such model can be retrieved from the present one with surplus-dependent premium rate by letting $c(u)=c+\varepsilon u$. See e.g. Cai and Dickson (2002), Gerber and Yang (2007) and Sundt and Teugels (1995).

As an illustration, for the remainder of this section, we assume a classical compound Poisson risk model with $k(t)=\lambda e^{-\lambda t}$ and $p_{t}(y)=p(y)$. Then, the integral equation (2.13) implies

$$
\varphi_{\delta, r}^{\prime}(u)=\frac{\lambda+\delta}{c+\varepsilon u} \varphi_{\delta, r}(u)-\frac{\lambda}{c+\varepsilon u} \int_{0}^{u} e^{-r(u-y)} p(y) d y-\frac{\lambda}{c+\varepsilon u} \int_{0}^{u} \varphi_{\delta, r}(u-y) p(y) d y .
$$

In general, it is not easy to solve $(5.1)$ for $\varphi_{\delta, r}(u)$. In the context of compound Poisson risk models with credit interest, integro-differential equation of the form (5.1) is usually transformed into a Volterra integral equation whose solution can be approximated recursively by Picard's sequence (see e.g. Cai and Dickson (2002) and Wu et al. (2007)). However, instead of inverting such a solution analytically with respect to $r$ to give $\tau_{\delta}(u, z)$ (which does not appear to be an easy task), an explicit expression for $\tau_{0}(u, z)$ is obtainable in the case of exponential claims using a direct method as follows.

Additionally we assume $p(y)=\mu e^{-\mu y}$. Then, omitting some straightforward algebra, application of the operator $(d / d u+\mu)$ to $(5.1)$ leads to the differential equation

$$
\varphi_{\delta, r}^{\prime \prime}(u)+\left(\mu+\frac{\varepsilon-\lambda-\delta}{c+\varepsilon u}\right) \varphi_{\delta, r}^{\prime}(u)-\frac{\delta \mu}{c+\varepsilon u} \varphi_{\delta, r}(u)=-\frac{\lambda \mu}{c+\varepsilon u} e^{-r u} .
$$

To solve the above equation for $\varphi_{\delta, r}(u)$, we further assume that $\delta=0$. Then (5.2) reduces to

$$
\varphi_{0, r}^{\prime \prime}(u)+\left(\mu+\frac{\varepsilon-\lambda}{c+\varepsilon u}\right) \varphi_{0, r}^{\prime}(u)=-\frac{\lambda \mu}{c+\varepsilon u} e^{-r u} .
$$

By Polyanin and Zaitsev (2003, Section 2.1.9 Solution 3), the solution to (5.3) is given by

$$
\varphi_{0, r}(u)=C_{1}+\int_{0}^{u} e^{-Q_{1}(x)}\left\{C_{2}+\int_{0}^{x} e^{Q_{1}(z)} Q_{2}(z) d z\right\} d x, \quad u \geq 0
$$

where

$$
Q_{1}(z)=\int_{0}^{z}\left(\mu+\frac{\varepsilon-\lambda}{c+\varepsilon v}\right) d v=\mu z+\left(1-\frac{\lambda}{\varepsilon}\right) \ln \left(1+\frac{\varepsilon}{c} z\right) \quad \text { and } \quad Q_{2}(z)=-\frac{\lambda \mu}{c+\varepsilon z} e^{-r z}
$$

and $C_{1}$ and $C_{2}$ are constants to be determined by two boundary conditions. The first boundary condition can be obtained by letting $u \rightarrow \infty$ in (5.4) together with $\lim _{u \rightarrow \infty} \varphi_{0, r}(u)=0$. This yields

$$
0=C_{1}+C_{2} \int_{0}^{\infty} e^{-Q_{1}(x)} d x+\int_{0}^{\infty} e^{-Q_{1}(x)} \int_{0}^{x} e^{Q_{1}(z)} Q_{2}(z) d z d x
$$

In addition, by putting $u=0$ into the integro-differential equation (5.1), one arrives at

$$
\varphi_{0, r}^{\prime}(0)=\frac{\lambda}{c} \varphi_{0, r}(0)
$$


which, according to (5.4), leads to the second boundary condition

$$
C_{2}=\frac{\lambda}{c} C_{1}
$$

Solving (5.6) and (5.7) simultaneously yields

$$
C_{1}=-\frac{\int_{0}^{\infty} e^{-Q_{1}(x)} \int_{0}^{x} e^{Q_{1}(z)} Q_{2}(z) d z d x}{1+\frac{\lambda}{c} \int_{0}^{\infty} e^{-Q_{1}(x)} d x} \quad \text { and } \quad C_{2}=-\frac{\lambda}{c} \frac{\int_{0}^{\infty} e^{-Q_{1}(x)} \int_{0}^{x} e^{Q_{1}(z)} Q_{2}(z) d z d x}{1+\frac{\lambda}{c} \int_{0}^{\infty} e^{-Q_{1}(x)} d x},
$$

and therefore (5.4) becomes

$\varphi_{0, r}(u)=-\left[\frac{1+\frac{\lambda}{c} \int_{0}^{u} e^{-Q_{1}(x)} d x}{1+\frac{\lambda}{c} \int_{0}^{\infty} e^{-Q_{1}(x)} d x}\right] \int_{0}^{\infty} e^{-Q_{1}(x)} \int_{0}^{x} e^{Q_{1}(z)} Q_{2}(z) d z d x+\int_{0}^{u} e^{-Q_{1}(x)} \int_{0}^{x} e^{Q_{1}(z)} Q_{2}(z) d z d x$.

From the above expression $\varphi_{0, r}(u)$ depends on $r$ only through $Q_{2}(z)$. By noting that

$$
\int_{0}^{u} e^{-Q_{1}(x)} \int_{0}^{x} e^{Q_{1}(z)} Q_{2}(z) d z d x=-\int_{0}^{u} e^{-r z}\left\{\int_{z}^{u} e^{-Q_{1}(x)} d x\right\} \frac{\lambda \mu}{c+\varepsilon z} e^{Q_{1}(z)} d z,
$$

inversion of (5.8) with respect to $r$ leads to

$$
\tau_{0}(u, z)=\left\{\left[\frac{1+\frac{\lambda}{c} \int_{0}^{u} e^{-Q_{1}(x)} d x}{1+\frac{\lambda}{c} \int_{0}^{\infty} e^{-Q_{1}(x)} d x}\right] \int_{z}^{\infty} e^{-Q_{1}(x)} d x-1(z<u) \int_{z}^{u} e^{-Q_{1}(x)} d x\right\} \frac{\lambda \mu}{c+\varepsilon z} e^{Q_{1}(z)} .
$$

A more explicit formula for $\tau_{0}(u, z)$ can be obtained by substituting into (5.9) the expression of $Q_{1}(z)$ given in (5.5). We then obtain

$$
\begin{aligned}
\tau_{0}(u, z)=\{ & {\left[\frac{1+\frac{\lambda}{c} \int_{0}^{u} e^{-\mu x}\left(1+\frac{\varepsilon}{c} x\right)^{-1+\frac{\lambda}{\varepsilon}} d x}{1+\frac{\lambda}{c} \int_{0}^{\infty} e^{-\mu x}\left(1+\frac{\varepsilon}{c} x\right)^{-1+\frac{\lambda}{\varepsilon}} d x}\right] \int_{z}^{\infty} e^{-\mu(x-z)}\left(\frac{c+\varepsilon x}{c+\varepsilon z}\right)^{-1+\frac{\lambda}{\varepsilon}} d x } \\
& \left.-1(z<u) \int_{z}^{u} e^{-\mu(x-z)}\left(\frac{c+\varepsilon x}{c+\varepsilon z}\right)^{-1+\frac{\lambda}{\varepsilon}} d x\right\} \frac{\lambda \mu}{c+\varepsilon z},
\end{aligned}
$$

which is expressed explicitly in terms of model parameters.

We remark that the joint density of the triplet $\left(U_{T^{-}},\left|U_{T}\right|, R_{N_{T}-1}\right)$ consists of two parts: $h_{1,0}$ in $(2.2)$ and $h_{2,0}$ in (2.3), where $h_{2,0}$ follows immediately from (5.10) together with (2.6) in Lemma 1.

\section{Extension of the approach: Absolute ruin model}

In fact, the general structure regarding the discounted densities and the generalized Gerber-Shiu function presented in Section 2 holds true more generally in the context of an absolute ruin model (see e.g. Cai (2007), Cai et al. (2009b), Dassios and Embrechts (1989), Gerber and Yang (2007) and Zhu and Yang (2008)). Under the absolute ruin model, the insurance company is allowed to continue its business even if its surplus drops below zero as long as the surplus is still above a (negative) critical level $u_{0}$. When the surplus level at time $t$ is $U_{t}^{\mathrm{A}}$ such that $u_{0}<U_{t}^{\mathrm{A}}<0$, the shortfall is subject to an instantaneous borrowing 
rate (or debit interest) of $\varepsilon_{d}\left(U_{t}^{\mathrm{A}}\right)$, where $\varepsilon_{d}($.$) is a positive function. By extending the surplus-dependent$ premium $c($.$) to negative surplus level, the critical level u_{0}$ is the largest root of the equation (in $\xi$ )

$$
c(\xi)+\varepsilon_{d}(\xi) \xi=0, \quad \xi<0 .
$$

See Cai et al. (2009b). If we define the quantity

$$
c^{\mathrm{A}}(u)= \begin{cases}c(u), & u \geq 0 \\ c(u)+\varepsilon_{d}(u) u, & u_{0}<u<0\end{cases}
$$

the surplus process $\left\{U_{t}^{\mathrm{A}}\right\}_{t \geq 0}$ satisfies

$$
d U_{t}^{\mathrm{A}}=c^{\mathrm{A}}\left(U_{t}^{\mathrm{A}}\right) d t-d S_{t}, \quad t \geq 0 .
$$

In addition to the technical conditions $\int_{u}^{x}\left[c^{\mathrm{A}}(v)\right]^{-1} d v<\infty$ for $x>u>u_{0}$ and $\int_{u}^{\infty}\left[c^{\mathrm{A}}(v)\right]^{-1} d v=\infty$ for $u>u_{0}$, we also assume that $\int_{u_{0}+\epsilon}^{u_{0}}\left[c^{\mathrm{A}}(v)\right]^{-1} d v=\infty$ for any $\epsilon>0$. See Gerber and Yang (2007). The time of ruin of the process $\left\{U_{t}^{\mathrm{A}}\right\}_{t \geq 0}$ is defined by $T^{\mathrm{A}}=\inf \left\{t \geq 0: U_{t}^{\mathrm{A}} \leq u_{0}\right\}$ with $T^{\mathrm{A}}=\infty$ if $U_{t}^{\mathrm{A}}>u_{0}$ for all $t \geq 0$. The generalized Gerber-Shiu function of our interest is

$$
m_{\delta}^{\mathrm{A}}(u)=E\left[e^{-\delta T^{\mathrm{A}}} w\left(U_{\left(T^{\mathrm{A}}\right)^{-}}^{\mathrm{A}},\left|U_{T^{\mathrm{A}}}^{\mathrm{A}}\right|, R_{N_{T^{\mathrm{A}}}-1}^{\mathrm{A}}\right) 1\left(T^{\mathrm{A}}<\infty\right) \mid U_{0}^{\mathrm{A}}=u\right], \quad u>u_{0},
$$

where the surplus prior to ruin $U_{\left(T^{\mathrm{A}}\right)^{-}}^{\mathrm{A}}$, deficit at ruin $\left|U_{T^{\mathrm{A}}}^{\mathrm{A}}\right|$ and the surplus immediately after the second last claim before ruin $R_{N_{T A}-1}^{\mathrm{A}}$ are defined accordingly. Furthermore, $\gamma^{\mathrm{A}}(u, t)$ and $\vartheta^{\mathrm{A}}(u, x)$ are given by (1.2) and (1.4) respectively with $c^{\mathrm{A}}($.$) in place of c($.$) . Analogous to (2.2) and (2.3), we define the$ discounted joint densities

$$
h_{1, \delta}^{\mathrm{A}}(x, y \mid u)=\frac{1}{c^{\mathrm{A}}(x)} e^{-\delta \vartheta^{\mathrm{A}}(u, x)} k\left(\vartheta^{\mathrm{A}}(u, x)\right) p_{\vartheta^{\mathrm{A}}(u, x)}(x+y), \quad x>u>u_{0} ; y>-u_{0},
$$

for ruin occurring on the first claim and

$$
h_{2, \delta}^{\mathrm{A}}(x, y, z \mid u)=\int_{0}^{\infty} e^{-\delta t} h_{2}^{\mathrm{A}}(t, x, y, z \mid u) d t, \quad x>z>u_{0} ; y>-u_{0},
$$

where $h_{2}^{\mathrm{A}}(t, x, y, z \mid u)$ is the joint density of $\left(T^{\mathrm{A}}, U_{\left(T^{\mathrm{A}}\right)^{-}}^{\mathrm{A}},\left|U_{T^{\mathrm{A}}}^{\mathrm{A}}\right|, R_{N_{T^{\mathrm{A}}}-1}^{\mathrm{A}}\right)$ at $(t, x, y, z)$ for ruin on subsequent claims given $U_{0}^{\mathrm{A}}=u$. Adapting Lemma 1 and Proposition 1 to the absolute ruin context, we have that

$$
h_{2, \delta}^{\mathrm{A}}(x, y, z \mid u)=\tau_{\delta}^{\mathrm{A}}(u, z) h_{1, \delta}^{\mathrm{A}}(x, y \mid z), \quad x>z>u_{0} ; y>-u_{0},
$$

and

$$
m_{\delta}^{\mathrm{A}}(u)=\beta_{\delta}^{\mathrm{A}}(u)+\int_{-u_{0}}^{\infty} \tau_{\delta}^{\mathrm{A}}(u, z) \beta_{\delta}^{\mathrm{A}}(z) d z, \quad u>u_{0}
$$

where

$$
\beta_{\delta}^{\mathrm{A}}(u)=\int_{-u_{0}}^{\infty} \int_{u}^{\infty} w(x, y, u) h_{1, \delta}^{\mathrm{A}}(x, y \mid u) d x d y, \quad u>u_{0} .
$$

Again, the generalized Gerber-Shiu function (6.1) is characterized by $\tau_{\delta}^{\mathrm{A}}(u, z)$ via $(6.2)$, and $\tau_{\delta}^{\mathrm{A}}(u, z)$ can be determined through its (two-sided) Laplace transform $\varphi_{\delta, r}^{\mathrm{A}}(u)=\int_{u_{0}}^{\infty} e^{-r z} \tau_{\delta}^{\mathrm{A}}(u, z) d z$ given by

$$
\varphi_{\delta, r}^{\mathrm{A}}(u)=\sum_{n=1}^{\infty} E\left[e^{-\delta \sum_{j=1}^{n} V_{j}} e^{-r R_{n}^{\mathrm{A}}} 1\left(R_{i}^{\mathrm{A}}>u_{0}, i=1,2, \ldots, n\right) \mid U_{0}^{\mathrm{A}}=u\right], \quad u>u_{0} .
$$


Then $\varphi_{\delta, r}^{\mathrm{A}}(u)$ can be obtained by conditioning on the time and the amount of the first claim

$$
\varphi_{\delta, r}^{\mathrm{A}}(u)=\int_{0}^{\infty} e^{-\delta t}\left\{\int_{0}^{\gamma^{\mathrm{A}}(u, t)-u_{0}}\left[e^{-r\left(\gamma^{\mathrm{A}}(u, t)-y\right)}+\varphi_{\delta, r}^{\mathrm{A}}\left(\gamma^{\mathrm{A}}(u, t)-y\right)\right] p_{t}(y) d y\right\} k(t) d t .
$$

In general, due to the presence of debit interest below level $0, \varphi_{\delta, r}^{\mathrm{A}}(u)$ is of different functional form depending on whether $u_{0}<u<0$ or $u \geq 0$. Therefore we shall denote

$$
\varphi_{\delta, r}^{\mathrm{A}}(u)=\left\{\begin{array}{ll}
\varphi_{\delta, r,+}^{\mathrm{A}}(u)=\int_{u_{0}}^{\infty} e^{-r z} \tau_{\delta,+}^{\mathrm{A}}(u, z) d z, & u \geq 0 \\
\varphi_{\delta, r,-}^{\mathrm{A}}(u)=\int_{u_{0}}^{\infty} e^{-r z} \tau_{\delta,-}^{\mathrm{A}}(u, z) d z, & u_{0}<u<0
\end{array} .\right.
$$

By continuity one also has

$$
\varphi_{\delta, r,+}^{\mathrm{A}}(0)=\varphi_{\delta, r,-}^{\mathrm{A}}\left(0^{-}\right)
$$

Furthermore, the boundary condition

$$
\varphi_{\delta, r,-}^{\mathrm{A}}\left(u_{0}^{+}\right)=0
$$

is a consequence of the additional technical assumption by Gerber and Yang (2007) since ruin is expected to occur upon the first claim with initial surplus $u_{0}^{+}$.

In order to exploit more structure, for the remainder of this section, we make the assumption that $c(.) \equiv c$. In our analysis various quantities, for instance, $\varphi_{\delta, r}(u) \phi_{\delta}, f_{\delta}(y)$ and $g_{\delta}(y)$ defined in Section 3 for the constant premium case, will be used. The positive security loading condition $c E[V]>E[Y]$ is assumed to hold.

Using the same argument used to obtain (3.3), one has the defective renewal equation for $\varphi_{\delta, r,+}^{\mathrm{A}}(u)$ given by

$$
\varphi_{\delta, r,+}^{\mathrm{A}}(u)=\phi_{\delta} \int_{0}^{u} \varphi_{\delta, r,+}^{\mathrm{A}}(u-y) f_{\delta}(y) d y+\varpi_{\delta, r}^{\mathrm{A}}(u), \quad u \geq 0
$$

where

$$
\varpi_{\delta, r}^{\mathrm{A}}(u)=\varphi_{\delta, r}(0) e^{-r u}+\phi_{\delta} \int_{0}^{u-u_{0}} e^{-r(u-y)} f_{\delta}(y) d y+\phi_{\delta} \int_{u}^{u-u_{0}} \varphi_{\delta, r,-}^{\mathrm{A}}(u-y) f_{\delta}(y) d y, \quad u \geq 0 .
$$

The solution to $(6.6)$ is

$$
\varphi_{\delta, r,+}^{\mathrm{A}}(u)=\frac{1}{1-\phi_{\delta}} \int_{0}^{u} \varpi_{\delta, r}^{\mathrm{A}}(u-y) g_{\delta}(y) d y+\varpi_{\delta, r}^{\mathrm{A}}(u), \quad u \geq 0 .
$$

The above solution for $\varphi_{\delta, r,+}^{\mathrm{A}}(u)$ depends on $\varphi_{\delta, r,-}^{\mathrm{A}}(u)$ via $\varpi_{\delta, r}^{\mathrm{A}}(u)$. The determination of $\varphi_{\delta, r,-}^{\mathrm{A}}(u)$ is usually done by making further assumptions on the interclaim time, the claim size and/or the debit interest function $\varepsilon_{d}($.$) .$

Here we assume a compound Poisson model with constant debit interest such that $k(t)=\lambda e^{-\lambda t}$, $p_{t}(y)=p(y)$ and $\varepsilon_{d}(.) \equiv \varepsilon_{d}$, and therefore $u_{0}=-c / \varepsilon_{d}$. As in Example 4, one can verify that a change of variables in (6.3) followed by differentiation leads to a piecewise integro-differential equation. One of the two pieces is given by

$$
\begin{array}{r}
\left(\varphi_{\delta, r,-}^{\mathrm{A}}\right)^{\prime}(u)=\frac{\lambda+\delta}{c+\varepsilon_{d} u} \varphi_{\delta, r,-}^{\mathrm{A}}(u)-\frac{\lambda}{c+\varepsilon_{d} u} \int_{0}^{u+\frac{c}{\varepsilon_{d}}} e^{-r(u-y)} p(y) d y-\frac{\lambda}{c+\varepsilon_{d} u} \int_{0}^{u+\frac{c}{\varepsilon_{d}}} \varphi_{\delta, r,-}^{\mathrm{A}}(u-y) p(y) d y \\
-\frac{c}{\varepsilon_{d}}<u \leq 0
\end{array}
$$


while the remaining piece can be transformed to (6.8).

By further assuming $p(y)=\mu e^{-\mu y}$ and $\delta=0$, application of the operator $(d / d u+\mu)$ to (6.9) leads to

$$
\left(\varphi_{0, r,-}^{\mathrm{A}}\right)^{\prime \prime}(u)+\left(\mu+\frac{\varepsilon_{d}-\lambda}{c+\varepsilon_{d} u}\right)\left(\varphi_{0, r,-}^{\mathrm{A}}\right)^{\prime}(u)=-\frac{\lambda \mu}{c+\varepsilon_{d} u} e^{-r u}, \quad-\frac{c}{\varepsilon_{d}}<u \leq 0,
$$

which is structurally identical to (5.3). Hence its general solution is given by

$$
\varphi_{0, r,-}^{\mathrm{A}}(u)=C_{3}+C_{4} \Gamma_{1}(u)+\Gamma_{2}(u), \quad-\frac{c}{\varepsilon_{d}}<u \leq 0,
$$

where

$$
\Gamma_{1}(u)=\int_{0}^{u} e^{-Q_{3}(x)} d x \quad \text { and } \quad \Gamma_{2}(u)=\int_{0}^{u} e^{-Q_{3}(x)} \int_{0}^{x} e^{Q_{3}(z)} Q_{4}(z) d z d x .
$$

Here $Q_{3}(z)$ and $Q_{4}(z)$ have the same definition as $Q_{1}(z)$ and $Q_{2}(z)$ respectively (as in (5.5)) but with $\varepsilon$ replaced by $\varepsilon_{d}$, and the constants $C_{3}$ and $C_{4}$ are determined by the boundary conditions (6.4) and (6.5). First, (6.5) implies

$$
C_{3}+C_{4} \Gamma_{1}\left(-\frac{c}{\varepsilon_{d}}\right)+\Gamma_{2}\left(-\frac{c}{\varepsilon_{d}}\right)=0
$$

Second, by noting from (6.11) that $\Gamma_{1}(0)=\Gamma_{2}(0)=0$, (6.4) together with (6.6) leads to

$$
\varpi_{0, r}^{\mathrm{A}}(0)=C_{3} .
$$

Utilizing the well-known results

$$
\phi_{0}=\frac{\lambda}{\mu c} \quad \text { and } \quad f_{0}(y)=\mu e^{-\mu y}, \quad y>0
$$

for exponential claims (see e.g. Gerber and Shiu (1998)), application of (6.7) to (6.13) yields

$$
\Theta_{r}+\frac{\lambda}{c} \int_{0}^{\frac{c}{\varepsilon_{d}}} \varphi_{0, r,-}^{\mathrm{A}}(-y) e^{-\mu y} d y=C_{3}
$$

where

$$
\Theta_{r}=\varphi_{0, r}(0)+\frac{\lambda}{c} \int_{0}^{\frac{c}{\varepsilon_{d}}} e^{r y} e^{-\mu y} d y
$$

Substitution of the solution form (6.10) into (6.15) followed by some algebraic manipulations gives

$$
\Theta_{r}+\frac{\lambda}{c} \int_{-\frac{c}{\varepsilon_{d}}}^{0} \Gamma_{2}(y) e^{\mu y} d y+C_{3}\left[\frac{\lambda}{\mu c}\left(1-e^{-\frac{\mu c}{\varepsilon_{d}}}\right)\right]+C_{4}\left\{\frac{\lambda}{c} \int_{-\frac{c}{\varepsilon_{d}}}^{0} \Gamma_{1}(y) e^{\mu y} d y\right\}=C_{3} .
$$

Solving (6.12) and (6.17) simultaneously yields

$$
C_{3}=\frac{1}{\Upsilon}\left\{\Gamma_{1}\left(-\frac{c}{\varepsilon_{d}}\right)\left[\Theta_{r}+\frac{\lambda}{c} \int_{-\frac{c}{\varepsilon_{d}}}^{0} \Gamma_{2}(y) e^{\mu y} d y\right]-\frac{\lambda}{c} \Gamma_{2}\left(-\frac{c}{\varepsilon_{d}}\right) \int_{-\frac{c}{\varepsilon_{d}}}^{0} \Gamma_{1}(y) e^{\mu y} d y\right\}
$$

and

$$
C_{4}=-\frac{1}{\Upsilon}\left\{\Gamma_{2}\left(-\frac{c}{\varepsilon_{d}}\right)\left[1-\frac{\lambda}{\mu c}\left(1-e^{-\frac{\mu c}{\varepsilon_{d}}}\right)\right]+\Theta_{r}+\frac{\lambda}{c} \int_{-\frac{c}{\varepsilon_{d}}}^{0} \Gamma_{2}(y) e^{\mu y} d y\right\}
$$


where

$$
\Upsilon=\Gamma_{1}\left(-\frac{c}{\varepsilon_{d}}\right)\left[1-\frac{\lambda}{\mu c}\left(1-e^{-\frac{\mu c}{\varepsilon_{d}}}\right)\right]+\frac{\lambda}{c} \int_{-\frac{c}{\varepsilon_{d}}}^{0} \Gamma_{1}(y) e^{\mu y} d y .
$$

Hence a complete characterization of $\varphi_{0, r,-}^{\mathrm{A}}(u)$ is given by (6.10) together with (6.18), (6.19) and (6.20). Then $\varphi_{0, r,+}^{\mathrm{A}}(u)$ can be determined by (6.8) together with (6.7) (at $\delta=0$ ). Note that the compound geometric density appearing in (6.8) can be obtained from (3.21) at $\delta=0$, i.e.

$$
g_{0}(y)=\frac{\lambda}{\mu c}\left(\mu-\frac{\lambda}{c}\right) e^{-\left(\mu-\frac{\lambda}{c}\right) y}, \quad y \geq 0 .
$$

To invert $\varphi_{0, r,-}^{\mathrm{A}}(u)$ with respect to $r$ to obtain $\tau_{0,-}^{\mathrm{A}}(u, z)$, we note from $(6.11)$ that $\Gamma_{1}(u)$ does not depend on $r$ while $\Gamma_{2}(u)$ depends on $r$ through $Q_{4}(z)$. Note also that $\Upsilon$ is independent of $r$, and therefore $C_{3}$ and $C_{4}$ given in (6.18) and (6.19) only depend on $r$ via $\Gamma_{2}(u)$ and $\Theta_{r}$. Using (3.12) with the fact that $\rho=0$ when $\delta=0,(6.16)$ can be rewritten as

$$
\Theta_{r}=\frac{\lambda}{c} \int_{0}^{\infty} e^{-r z} d z+\frac{\lambda}{c} \int_{-\frac{c}{\varepsilon_{d}}}^{0} e^{-r z} e^{\mu z} d z
$$

On the other hand, by changing the order of integration, one has

$$
\Gamma_{2}(u)=-\int_{u}^{0} e^{-r z}\left\{\int_{u}^{z} e^{-Q_{3}(x)} d x\right\} \frac{\lambda \mu}{c+\varepsilon_{d} z} e^{Q_{3}(z)} d z,
$$

and therefore

$$
\int_{-\frac{c}{\varepsilon_{d}}}^{0} \Gamma_{2}(y) e^{\mu y} d y=-\int_{-\frac{c}{\varepsilon_{d}}}^{0} e^{-r z}\left\{\int_{-\frac{c}{\varepsilon_{d}}}^{z} e^{\mu y} \int_{y}^{z} e^{-Q_{3}(x)} d x d y\right\} \frac{\lambda \mu}{c+\varepsilon_{d} z} e^{Q_{3}(z)} d z .
$$

Applying (6.22), (6.23) and (6.24) to (6.18) and (6.19), one arrives at

$$
C_{3}=\frac{\lambda}{c \Upsilon} \Gamma_{1}\left(-\frac{c}{\varepsilon_{d}}\right) \int_{0}^{\infty} e^{-r z} d z+\int_{-\frac{c}{\varepsilon_{d}}}^{0} e^{-r z} \pi_{1}(z) d z
$$

and

$$
C_{4}=-\frac{\lambda}{c \Upsilon} \int_{0}^{\infty} e^{-r z} d z+\int_{-\frac{c}{\varepsilon_{d}}}^{0} e^{-r z} \pi_{2}(z) d z
$$

where

$$
\begin{aligned}
\pi_{1}(z)=\frac{\lambda}{c \Upsilon}\{ & \Gamma_{1}\left(-\frac{c}{\varepsilon_{d}}\right)\left[e^{\mu z}-\left(\int_{-\frac{c}{\varepsilon_{d}}}^{z} e^{\mu y} \int_{y}^{z} e^{-Q_{3}(x)} d x d y\right) \frac{\lambda \mu}{c+\varepsilon_{d} z} e^{Q_{3}(z)}\right] \\
& \left.+\left(\int_{-\frac{c}{\varepsilon_{d}}}^{z} e^{-Q_{3}(x)} d x\right) \frac{\lambda \mu}{c+\varepsilon_{d} z} e^{Q_{3}(z)}\left(\int_{-\frac{c}{\varepsilon_{d}}}^{0} \Gamma_{1}(y) e^{\mu y} d y\right)\right\}, \quad-\frac{c}{\varepsilon_{d}}<z<0,
\end{aligned}
$$

and

$$
\begin{aligned}
\pi_{2}(z)= & \frac{1}{\Upsilon}\left(\int_{-\frac{c}{\varepsilon_{d}}}^{z} e^{-Q_{3}(x)} d x\right) \frac{\lambda \mu}{c+\varepsilon_{d} z} e^{Q_{3}(z)}\left[1-\frac{\lambda}{\mu c}\left(1-e^{-\frac{\mu c}{\varepsilon_{d}}}\right)\right] \\
& +\frac{\lambda}{c \Upsilon}\left[\left(\int_{-\frac{c}{\varepsilon_{d}}}^{z} e^{\mu y} \int_{y}^{z} e^{-Q_{3}(x)} d x d y\right) \frac{\lambda \mu}{c+\varepsilon_{d} z} e^{Q_{3}(z)}-e^{\mu z}\right], \quad-\frac{c}{\varepsilon_{d}}<z<0 .
\end{aligned}
$$


Substitution of (6.23), (6.25) and (6.26) into (6.10) leads to

$$
\begin{aligned}
\varphi_{0, r,-}^{\mathrm{A}}(u)= & \frac{\lambda}{c \Upsilon}\left[\Gamma_{1}\left(-\frac{c}{\varepsilon_{d}}\right)-\Gamma_{1}(u)\right] \int_{0}^{\infty} e^{-r z} d z+\int_{-\frac{c}{\varepsilon_{d}}}^{0} e^{-r z}\left[\pi_{1}(z)+\Gamma_{1}(u) \pi_{2}(z)\right] d z \\
& -\int_{u}^{0} e^{-r z}\left\{\int_{u}^{z} e^{-Q_{3}(x)} d x\right\} \frac{\lambda \mu}{c+\varepsilon_{d} z} e^{Q_{3}(z)} d z, \quad-\frac{c}{\varepsilon_{d}}<u \leq 0 .
\end{aligned}
$$

Clearly, by inversion of (6.27) with respect to $r$, we obtain, for $-c / \varepsilon_{d}<u \leq 0$,

$$
\tau_{0,-}^{\mathrm{A}}(u, z)= \begin{cases}\frac{\lambda}{c \Upsilon}\left[\Gamma_{1}\left(-\frac{c}{\varepsilon_{d}}\right)-\Gamma_{1}(u)\right], & z \geq 0 \\ \pi_{1}(z)+\Gamma_{1}(u) \pi_{2}(z)-1(z>u)\left\{\int_{u}^{z} e^{-Q_{3}(x)} d x\right\} \frac{\lambda \mu}{c+\varepsilon_{d} z} e^{Q_{3}(z)} . & -\frac{c}{\varepsilon_{d}}<z<0\end{cases}
$$

which gives an expression for $\tau_{0,-}^{\mathrm{A}}(u, z)$.

Next, to obtain $\tau_{0,+}^{\mathrm{A}}(u, z)$, we make use of $(3.13),(6.14)$ and $\varphi_{0, r,-}^{\mathrm{A}}(u)=\int_{-c / \varepsilon_{d}}^{\infty} e^{-r z} \tau_{0,-}^{\mathrm{A}}(u, z) d z$ so that (6.7) with $\delta=0$ can be represented as

$$
\begin{aligned}
\varpi_{0, r}^{\mathrm{A}}(u) & =\frac{\lambda}{c} \int_{u}^{\infty} e^{-r z} d z+\frac{\lambda}{c} \int_{-\frac{c}{\varepsilon_{d}}}^{u} e^{-r z} e^{-\mu(u-z)} d z+\frac{\lambda}{c} \int_{-\frac{c}{\varepsilon_{d}}}^{\infty} e^{-r z} \int_{-\frac{c}{\varepsilon_{d}}}^{0} e^{-\mu(u-y)} \tau_{0,-}^{\mathrm{A}}(y, z) d y d z \\
& =\int_{-\frac{c}{\varepsilon_{d}}}^{\infty} e^{-r z} \sigma^{\mathrm{A}}(u, z) d z, \quad u \geq 0
\end{aligned}
$$

where

$$
\sigma^{\mathrm{A}}(u, z)=\frac{\lambda}{c}\left\{\int_{-\frac{c}{\varepsilon_{d}}}^{0} e^{-\mu(u-y)} \tau_{0,-}^{\mathrm{A}}(y, z) d y+1(z<u) e^{-\mu(u-z)}+1(z \geq u)\right\}, \quad u \geq 0 ; z>-\frac{c}{\varepsilon_{d}} .
$$

By putting (6.29) into (6.8) with the use of (6.21), inversion of the resulting expression with respect to $r$ results in

$$
\tau_{0,+}^{\mathrm{A}}(u, z)=\frac{\lambda}{c} \int_{0}^{u} \sigma^{\mathrm{A}}(u-y, z) e^{-\left(\mu-\frac{\lambda}{c}\right) y} d y+\sigma^{\mathrm{A}}(u, z), \quad u \geq 0 ; z>-\frac{c}{\varepsilon_{d}}
$$

which is an expression of $\tau_{0,+}^{\mathrm{A}}(u, z)$ in terms of $\tau_{0,-}^{\mathrm{A}}(u, z)$ given by $(6.28)$.

\section{Concluding remarks}

In this paper, the generalized Gerber-Shiu function proposed by Cheung et al. (2010a) is considered in a general Sparre Andersen risk model with surplus-dependent premium rate. It is shown that under the present model, the generalized Gerber-Shiu function $m_{\delta}(u)$ can be characterized by a transition function $\tau_{\delta}(u, z)$ which is independent of the penalty function. The determination of $\tau_{\delta}(u, z)$ can be done through its Laplace transform $\varphi_{\delta, r}(u)=\int_{0}^{\infty} e^{-r z} \tau_{\delta}(u, z) d z$, which is in turn evaluated by additional assumptions on the premium rate function $c($.$) , claim size and/or interclaim time distributions. The$ evaluation of $\varphi_{\delta, r}(u)$ (or its special case with $\delta=0$ ) is demonstrated in various risk models with (i) constant premium; (ii) a threshold dividend strategy; (iii) credit interest; or (iv) debit interest. In most 
cases analytic Laplace transform inversion of $\varphi_{\delta, r}(u)$ with respect to $r$ (to obtain $\tau_{\delta}(u, z)$ ) is performed, leading to a complete characterization of the generalized Gerber-Shiu function of our interest according to Proposition 1. The approach presented in this paper is different from the standard approach (which usually involves conditioning on the time and the amount of the first claim to obtain an integral and/or integro-differential equation for the Gerber-Shiu function itself), and we do not have to assume any specific form or differentiability of the penalty function.

As far as the discounted joint distribution of the triplet $\left(U_{T^{-}},\left|U_{T}\right|, R_{N_{T}-1}\right)$ is concerned, it is instructive to note that it can be readily obtained from $\tau_{\delta}(u, z)$ via Lemma 1 . This is also different from the usual approach in which the generalized Gerber-Shiu function $m_{\delta}(u)$ is evaluated with the choice of penalty function $w(x, y, z)=e^{-s_{1} x-s_{2} y-s_{3} z}$ and then inverted with respect to $\left(s_{1}, s_{2}, s_{3}\right)$. Our approach has the advantage that we only have to perform Laplace transform inversion with respect to one argument (to obtain $\tau_{\delta}(u, z)$ from $\left.\varphi_{\delta, r}(u)\right)$ instead of three in the usual approach.

Finally, we also remark that a matrix extension of the general structure exploited in Section 2 is possible by employing the ideas in Cheung and Landriault (2009).

\section{References}

[1] Albrecher, Hansjörg and Jef L. Teugels. 2006. Exponential behavior in the presence of dependence in risk theory. Journal of Applied Probability 43(1): 257-273.

[2] Badescu, Andrei L., Eric C.K. Cheung and David Landriault. 2009. Dependent risk models with bivariate phase-type distributions. Journal of Applied Probability 46(1): 113-131.

[3] Boudreault, Mathieu, Helene Cossette, David Landriault and Etienne Marceau. 2006. On a risk model with dependence between interclaim arrivals and claim sizes. Scandinavian Actuarial Journal 2006(5): 265-285.

[4] Bühlmann, Hans. 1970. Mathematical Methods in Risk Theory. New York: Springer.

[5] Cai, Jun. 2007. On the time value of absolute ruin with debit interest. Advances in Applied Probability 39(2): 343-359.

[6] Cai, Jun And David C.M. Dickson. 2002. On the expected discounted penalty function at ruin of a surplus process with interest. Insurance: Mathematics and Economics 30(3): 389-404.

[7] Cai, Jun, Runhuan Feng and Gordon E. Willmot. 2009a. The compound Poisson surplus model with interest and liquid reserves: Analysis of the Gerber-Shiu discounted penalty function. Methodology and Computing in Applied Probability 11(3): 401-423.

[8] Cai, Jun, Runhuan Feng and Gordon E. Willmot. 2009b. On the total discounted operating costs up to default and its applications. Advances in Applied Probability 41(2): 495-522.

[9] Cheung, Eric C.K. and David Landriault. 2009. Analysis of a generalized penalty function in a semi-Markovian risk model. North American Actuarial Journal 13(4): 497-513.

[10] Cheung, Eric, C.K. and David Landriault. 2010. A generalized penalty function with the maximum surplus prior to ruin in a MAP risk model. Insurance: Mathematics and Economics 46(1): 127-134. 
[11] Cheung, Eric C.K., David Landriault, Gordon E. Willmot and Jae-Kyung Woo. 2010 a. Gerber-Shiu analysis with a generalized penalty function. Scandinavian Actuarial Journal 2010(3): 185-199.

[12] Cheung, Eric C.K., David Landriault, Gordon E. Willmot and Jae-Kyung Woo. $2010 b$. Structural properties of Gerber-Shiu functions in dependent Sparre Andersen models. Insurance: Mathematics and Economics 46(1): 117-126.

[13] Cheung, Eric C.K., David Landriault, Gordon E. Willmot and Jae-Kyung Woo. 2011. On orderings and bounds in a generalized Sparre Andersen risk model. Applied Stochastic Models in Business and Industry. In press.

[14] Cossette, Helene, Etienne Marceau and Fouad Marri. 2008. On the compound Poisson risk model with dependence based on a generalized Farlie-Gumbel-Morgenstern copula. Insurance: Mathematics and Economics 43(3): 444-455.

[15] Dassios, Angelos and Paul Embrechts. 1989. Martingales and insurance risk. Communications in Statistics - Stochastic Models 5(2): 181-217.

[16] Dickson, David C.M. And Christian Hipp. 2001. On the time to ruin for Erlang(2) risk processes. Insurance: Mathematics and Economics 29(3): 333-344.

[17] Embrechts, Paul and Hanspeter Schmidli. 1994. Ruin estimation for a general insurance risk model. Advances in Applied Probability 26(2): 404-422.

[18] Feng, Runhuan. 2009. On the total operating costs up to default in a renewal risk model. Insurance: Mathematics and Economics 45(2): 305-314.

[19] Gerber, Hans U. and Elias S.W. Shiu. 1998. On the time value of ruin. North American Actuarial Journal 2(1): 48-72.

[20] Gerber, Hans U. and Elias S.W. Shiu. 2005. The time value of ruin in a Sparre Andersen model. North American Actuarial Journal 9(2): 49-69.

[21] Gerber, Hans U. and Elias S.W. Shiu. 2006. On optimal dividend strategies in the compound Poisson model. North American Actuarial Journal 10(2): 76-93.

[22] Gerber, Hans U. and Hailiang Yang. 2007. Absolute ruin probabilities in a jump diffusion risk model with investment. North American Actuarial Journal 11(3): 159-169.

[23] Lakshmikantham, Vangipuram and Rama Mohana M. Rao. 1995. Theory of IntegroDifferential Equations. Lausanne, Switzerland: Gordon and Breach Science Publishers.

[24] Landriault, David. 2008. Constant dividend barrier in a risk model with interclaim-dependent claim sizes. Insurance: Mathematics and Economics 42(1): 31-38.

[25] Landriault, David, And Gordon E. Willmot. 2008. On the Gerber-Shiu discounted penalty function in the Sparre Andersen model with an arbitrary interclaim time distribution. Insurance: Mathematics and Economics 42(2): 600-608.

[26] Li, Shuanming and Jose Garrido. 2004. On a class of renewal risk models with a constant dividend barrier. Insurance: Mathematics and Economics 35(3): 691-701. 
[27] Lin, Sheldon X. And Kristina P. Pavlova. 2006. The compound Poisson risk model with a threshold dividend strategy. Insurance: Mathematics and Economics 38(1): 57-80.

[28] Lin, Sheldon X. And Kristina P. Sendova. 2008. The compound Poisson risk model with multiple thresholds. Insurance: Mathematics and Economics 42(2): 617-627.

[29] Polyanin, Andrei D. And Valentin F. Zaitsev 2003. Handbook of Exact Solutions for Ordinary Differential Equations. New York: CRC Press.

[30] Resnick, Sidney I. 1992. Adventures in Stochastic Processes. Boston: Birkhauser.

[31] Sundt, Buørn. And Jozef L. Teugels. 1995. Ruin estimates under interest force. Insurance: Mathematics and Economics 16(1): 7-22.

[32] Willmot, Gordon E. 2007. On the discounted penalty function in the renewal risk model with general interclaim times. Insurance: Mathematics and Economics 41(1): 17-31.

[33] Willmot, Gordon E. And Jae-Kyung Woo. 2010. Surplus analysis for a class of Coxian interclaim time distributions with applications to mixed Erlang claim amounts. Insurance: Mathematics and Economics 46(1): 32-41.

[34] Woo, Jae-Kyung. 2010. Gerber-Shiu Analysis in some Dependent Sparre Andersen Risk Models. Ph.D. Thesis, University of Waterloo.

[35] Wu, Rong, Yuhua Lu and Ying Fang. 2007. On the Gerber-Shiu discounted penalty function for the ordinary renewal risk model with constant interest. North American Actuarial Journal 11(2): 119-134.

[36] Zhou, Xiaowen. 2004. When does surplus reach a certain level before ruin? Insurance: Mathematics and Economics 35(3): 553-561.

[37] Zhu, Jinxia and Hailiang Yang. 2008. Estimates for the absolute ruin probability in the compound Poisson risk model with credit and debit interest. Journal of Applied Probability 45(3): 818830 . 